\title{
Multidimensional Quasi-Eigenfunction Approximations and Multicomponent AM-FM Models
}

\author{
Joseph P. Havlicek, Senior Member, IEEE, David S. Harding, Student Member, IEEE, and \\ Alan Conrad Bovik, Fellow, IEEE
}

\begin{abstract}
We develop multicomponent AM-FM models for multidimensional signals. The analysis is cast in a general $n$-dimensional framework where the component modulating functions are assumed to lie in certain Sobolev spaces. For both continuous and discrete LSI systems with AM-FM inputs, powerful new approximations are introduced that provide closed form expressions for the responses in terms of the input modulations. The approximation errors are bounded by generalized energy variances quantifying the localization of the filter impulse response and by Sobolev norms quantifying the smoothness of the modulations. The approximations are then used to develop novel spatially localized demodulation algorithms that estimate the AM and FM functions for multiple signal components simultaneously from the channel responses of a multiband linear filterbank used to isolate components. Two discrete computational paradigms are presented. Dominant component analysis estimates the locally dominant modulations in a signal, which are useful in a variety of machine vision applications, while channelized components analysis delivers a true multidimensional multicomponent signal representation. We demonstrate the techniques on several images of general interest in practical applications, and obtain reconstructions that establish the validity of characterizing images of this type as sums of locally narrowband modulated components.
\end{abstract}

Index Terms-AM-FM models, amplitude modulation, approximation theory, channel bank filters, frequency modulation, image demodulation, image modulation models, image representation, multicomponent models.

\section{INTRODUCTION}

$\mathbf{I}$ $\mathrm{N}$ THIS paper, we develop new techniques for representing multidimensional signals in terms of their nonstationary structure. This is in contrast to the classical Fourier representation, which is a composition of stationary sinusoidal components each with an amplitude and frequency that are constant. Hence, with the Fourier transform, nonstationary

Manuscript received July 21, 1997; revised April 19, 1999. This research was supported in part by the Army Research Office under Contract DAAH 049510494 and by the Air Force Office of Scientific Research, Air Force Systems Command, USAF, under Grant F49620-93-1-0307. The associate editor coordinating the review of this manuscript and approving it for publication was Dr. Fabrice Heitz.

J. Havlicek is with the School of Electrical and Computer Engineering, The University of Oklahoma, Norman, OK 73019-1023 USA (e-mail: joebob@tobasco.ecn.ou.edu).

D. S. Harding and A. C. Bovik are with the Center for Vision and Image Sciences, The University of Texas, Austin, TX 78712-1084 USA (e-mail: dave@vision.ece.utexas.edu; bovik@ece.utexas.edu).

Publisher Item Identifier S 1057-7149(00)01262-8. structure can only be created by constructive and destructive interference between stationary components. In many engineering applications, however, nonstationary variations in local signal quantities such as the instantaneous amplitude and instantaneous frequency are information rich. Thus, an advantage can be gained by obtaining representations directly in terms of the nonstationarities. This is a primary impetus for performing time-frequency and wavelet analyses.

Recently, there has been a growing interest in techniques that model signals in terms of quasisinusoidal AM-FM functions which admit nonstationary amplitude and frequency modulations. Such AM-FM functions have been used successfully in the study of nonstationary one-dimensional (1-D) signals including human speech [1]-[5], as well as in the study of images and other multidimensional signals [6]-[12]. It is well known that joint amplitude and frequency modulations can be computed most accurately for signals having instantaneous frequency content that is highly coherent on a spatially local basis [2], [3], [8], [11], [13]-[15]. Furthermore, such locally narrowband signals are precisely the ones for which instantaneous amplitude and frequency are the most physically meaningful. Since the complicated nonstationary signals frequently encountered in practical applications do not generally admit representation as a single locally coherent AM-FM function, it is desirable to model these signals instead as multicomponent sums of locally narrowband AM-FM functions. However, this is a difficult problem and relatively few multicomponent AM-FM techniques have been reported.

In 1-D, the energy demodulation of mixtures (EDM) algorithm has been applied to demodulate synthetic two-component signals [16], and an iterative method involving the Teager-Kaiser energy operator (TKEO) has been used to track and demodulate multiple narrowband formants in human speech [4]. Two interesting multicomponent parametric methods have also emerged recently for the 1-D case: the model-based demodulation algorithm (MBDA), which is based on an extended Kalman filter, was used to demodulate multiple formants in human speech [5], while a multicomponent maximum likelihood estimation technique was demonstrated on synthetic two-component chirp signals in [17]. For multidimensional signals, the tracked multicomponent analysis (TMCA) approach reported in [6], [18] is the only previous multicomponent AM-FM technique of which we are aware. 
This paper studies the problem of analyzing a complex-valued signal $t: \mathbb{R}^{n} \rightarrow \mathbb{C}$ against the multidimensional, multicomponent AM-FM model

$$
t(\boldsymbol{x})=\sum_{i=1}^{K} a_{i}(\boldsymbol{x}) \exp \left[j \varphi_{i}(\boldsymbol{x})\right]=\sum_{i=1}^{K} t_{i}(\boldsymbol{x})
$$

where

$$
\begin{array}{ll}
K & \in \mathbb{N} ; \\
a_{i} & \mathbb{R}^{n} \rightarrow[0, \infty) ; \\
\varphi_{i} & \mathbb{R}^{n} \rightarrow \mathbb{R} .
\end{array}
$$

We also consider the corresponding discrete problem along with discrete implementations. In (1), we define the instantaneous amplitude of component $t_{i}(\boldsymbol{x})$ to be the function $a_{i}(\boldsymbol{x})$; it is alternatively known as the amplitude modulation function of $t_{i}(\boldsymbol{x})$. For two-dimensional (2-D) images, $a_{i}(\boldsymbol{x})$ may be interpreted as the contrast function of $t_{i}(\boldsymbol{x})$. By definition, the multidimensional instantaneous frequency of the (complex-valued) component $t_{i}(\boldsymbol{x})$ is the vector $\nabla \varphi_{i}(\boldsymbol{x})$ [15]. We alternatively call $\nabla \varphi_{i}(\boldsymbol{x})$ the frequency modulation function of $t_{i}(\boldsymbol{x})$. For 2-D images, $\nabla \varphi_{i}(x)$ embodies local texture orientation and coarseness. While the model (1) is complex-valued, many applications are concerned exclusively with real signals. To analyze a real signal $s(x)$ against (1), it is necessary to compute a complex extension $t(\boldsymbol{x})$ such that $\operatorname{Re}[t(\boldsymbol{x})]=s(\boldsymbol{x})$. Our technique for doing this is described in Section II.

For the remainder of this section, we will drop the subscripts from the component modulating functions in (1) in the interest of notational brevity. Let $\boldsymbol{x}=\left[\begin{array}{llll}x_{1} & x_{2} & \cdots & x_{n}\end{array}\right]^{T}$, and use the notation $a_{x_{i}}(x)$ to denote partial differentiation of a modulating function with respect to $x_{i}$. We quantify the coherency of each component $t_{i}(\boldsymbol{x})$ in (1) by the smoothness of the AM and FM functions expressed as functionals $\overline{\mathcal{D}^{q}}(a)=\left\|\mathcal{D}_{i}^{q}(a)\right\|_{\ell^{q}}$ and $\overline{\mathcal{S}^{q}}(\varphi)=\left\|\mathcal{S}_{i, j}^{q}(\varphi)\right\|_{\ell q}$, where

$$
\mathcal{D}_{i}^{q}(a)=\left[\int_{\mathbb{R}^{n}}\left|a_{x_{i}}(\boldsymbol{x})\right|^{q} d \boldsymbol{x}\right]^{1 / q}
$$

and

$$
\mathcal{S}_{i, j}^{q}(\varphi)=\left[\int_{\mathbb{R}^{n}}\left|\varphi_{x_{i}, x_{j}}(\boldsymbol{x})\right|^{q} d \boldsymbol{x}\right]^{1 / q}
$$

are Sobolev $q$-norms of orders one and two. For the functionals $\overline{\mathcal{D}^{q}}(a)$ and $\overline{\mathcal{S}^{q}}(\varphi)$, which simultaneously quantify the smoothness of the modulating functions in all directions, we employ the usual definition of the $\ell^{q}$-norm; viz.,

$$
\overline{\mathcal{S}^{q}}(\varphi)=\left[\sum_{i, j=1}^{n}\left|\mathcal{S}_{i, j}^{q}(\varphi)\right|^{q}\right]^{1 / q}
$$

Throughout the paper, we assume that the AM and FM functions in (1) and their first partials lie in appropriate Sobolev spaces so that the norms (2) and (3) exist and take finite values. Without loss of generality, we also assume that the AM functions are positive semidefinite.

For the discrete case, we impose two additional smoothness constraints. Let $t_{i}(\boldsymbol{k}): \mathbb{Z}^{n} \rightarrow \mathbb{C}$ contain the samples of a component $t_{i}(\boldsymbol{x})$ in (1), where $t_{i}(\boldsymbol{k})=a(\boldsymbol{k}) e^{j \varphi(\boldsymbol{k})}$ and $k=\left[\begin{array}{llll}k_{1} & k_{2} & \cdots & k_{n}\end{array}\right]^{T}$. Again without loss of generality, we assume a unity sampling interval throughout. The discrete functions $a(\boldsymbol{k}): \mathbb{Z}^{n} \rightarrow[0, \infty)$ and $\varphi(\boldsymbol{k}): \mathbb{Z}^{n} \rightarrow \mathbb{R}$ contain the samples of their continuous-domain counterparts. We write $\nabla \varphi(\boldsymbol{k})$ to denote the samples of the continuous-domain FM function $\nabla \varphi(\boldsymbol{x})$. The following definition will prove useful in formulating the constraints.

Definition: For $s \in \mathbb{R}$, denote by $P^{m}$ the set of connected paths (i.e., trajectories) $\sigma(s)=\left[\begin{array}{llll}\sigma_{1}(s) & \sigma_{2}(s) & \cdots & \sigma_{n}(s)\end{array}\right]^{T}$ through $\mathbb{R}^{n}$ or any connected subset of $\mathbb{R}^{n}$ for which each $\sigma_{i}(s)$ is a polynomial in $s$ of degree $m$ or less. Thus, $P^{1}$ is the set of all straight lines and line segments through $\mathbb{R}^{n}$.

For the AM functions in (1), we require that the smoothness functional

$$
\mathfrak{L}^{1}(a)=\sup _{\boldsymbol{\sigma} \in P^{1}}\left|\int_{\boldsymbol{\sigma}} \nabla a(\boldsymbol{x}) \cdot d \boldsymbol{x}\right|
$$

which is the supremum of the magnitudes of all line integrals of $\nabla a(\boldsymbol{x})$ along straight lines and line segments in $\mathbb{R}^{n}$, be finite. In the 1 -D case $(n=1), \mathfrak{L}^{1}(a) \leq \mathcal{D}_{1}^{1}(a)$. Similarly, for the FM functions we require that the smoothness functionals

$$
\mathfrak{S}_{i}^{1}(\varphi)=\sup _{\boldsymbol{\sigma} \in P^{1}} \int_{\boldsymbol{\sigma}}\left|\nabla \varphi_{x_{i}}(\boldsymbol{x})\right| d s
$$

be finite. Note that $\mathfrak{S}_{i}^{1}(\varphi)$ is the supremum of all path integrals of the magnitude of $\nabla \varphi_{x_{i}}(\boldsymbol{x})$ along straight lines and line segments in $\mathbb{R}^{n}$. In the 1-D case, $\mathfrak{S}_{i}^{1}(\varphi)$ is identical to the Sobolev $\operatorname{norm} \mathcal{S}_{1,1}^{1}(\varphi)$.

In Section II, we introduce new multidimensional quasi-eigenfunction approximations (QEA's) for the responses of linear shift invariant (LSI) systems to $n \mathrm{D}$ AM-FM inputs and develop tight bounds on the approximation errors. Analogous approximations have been studied previously for the case of 1-D continuous and discrete signals [3], [14] and $2-\mathrm{D}$ continuous signals [11]. The $n \mathrm{D}$ discrete theory presented here is both new and distinctive. For the continuous case, a new $n \mathrm{D}$ error bound is presented that contains an interesting dimensionally dependent term not previously revealed. We use the new QEA's to derive novel nonlinear $n \mathrm{D}$ AM-FM demodulation algorithms.

In Section IV, we introduce two new computational techniques that formulate estimates of the unknown AM and FM functions in (1). Both techniques begin by analyzing the signal $t(\boldsymbol{x})$ with a multiband linear filterbank. For each point in the signal, dominant component analysis (DCA) uses the filterbank channel responses to estimate the instantaneous amplitude and frequency of the component that dominates the local signal spectrum. Thus, it delivers as output only a single pair of modulating functions. Channelized components analysis (CCA) is a multicomponent technique that seeks to estimate simultaneously the modulating functions of the multiple components in an $n \mathrm{D}$ signal. The difference between CCA and TMCA is in how they decompose $t(\boldsymbol{x})$ into components [note that the component-wise decomposition indicated in (1) is not unique]. In CCA, modulating functions for one component are estimated from each filterbank channel response. Thus, with CCA, the filterbank channels are assumed to isolate components on a 
global scale. By contrast, in TMCA the instantaneous frequency of each individual component is permitted to lie in different channels at different points and Kalman filters are used to track the modulating functions of each component across the filterbank channel responses [6].

At present, a standardized method for evaluating the performance of computed AM-FM modeling techniques does not exist. In many of the previous studies, analysis was limited to synthetic signals [1], [3], [7], [10], [14], [16], [17], making it possible to compare the computed modulations with the analytical model used to generate the test signals. In other cases, AM-FM demodulation was applied to human speech or natural images and the computed modulations were subjectively compared with nonstationary structures in the original signals [2], [4], [8], [11]. We believe that the best way to evaluate computed AM-FM modeling techniques is to first reconstruct the signal by substituting the computed modulations back into, e.g., (1), and subsequently check for perceptual or quantitative agreement between the reconstruction and the original signal. This approach was used to evaluate the performance of MBDA in [5] and [19] and the performance of TMCA in [6], [18], and [20]. The question of whether quantitative or perceptual criteria are more appropriate for characterizing the extent to which the reconstructions and originals agree is application dependent and remains unanswered in general.

\section{COMPLEX EXTENSION OF ReAL-VALUed Signals}

The instantaneous amplitude and phase of any complex component $t_{i}: \mathbb{R}^{n} \rightarrow \mathbb{C}$ are unique. The instantaneous frequency is also therefore unique. For a real component $s_{i}: \mathbb{R}^{n} \rightarrow \mathbb{R}$, however, the instantaneous amplitude, phase, and frequency are ambiguous. In fact, for any given real component there are infinitely many distinct pairs of functions $a_{i}(\boldsymbol{x})$ and $\varphi_{i}(\boldsymbol{x})$ that satisfy $s_{i}(\boldsymbol{x})=a_{i}(\boldsymbol{x}) \cos \left[\varphi_{i}(\boldsymbol{x})\right]$. Thus, adding an imaginary part to $s_{i}(\boldsymbol{x})$ is equivalent to selecting one particular pair of modulating functions $a_{i}(\boldsymbol{x})$ and $\nabla \varphi_{i}(\boldsymbol{x})$ to associate with $s_{i}(\boldsymbol{x})$. We note in passing that any method which computes AM and FM functions from the real values $s_{i}(\boldsymbol{x})$ alone (such as the TKEO, for example) may conversely be interpreted as implicitly specifying an imaginary part $j a_{i}(\boldsymbol{x}) \sin \left[\varphi_{i}(\boldsymbol{x})\right]$.

Let $s(\boldsymbol{x})=\sum_{i=1}^{K} s_{i}(\boldsymbol{x})$, where $s_{i}(\boldsymbol{x})=a_{i}(\boldsymbol{x}) \cos \left[\varphi_{i}(\boldsymbol{x})\right]$ and where the modulating functions $a_{i}(\boldsymbol{x})$ and $\nabla \varphi_{i}(\boldsymbol{x})$ are unknown and nonunique. Before $s(\boldsymbol{x})$ can be analyzed against (1), a complex extension must be formulated by adding an imaginary part. If the imaginary part is computed from $s(\boldsymbol{x})$ using a linear operator, then it is precisely equal to $j \sum_{i=1}^{K} a_{i}(\boldsymbol{x}) \sin \left[\varphi_{i}(\boldsymbol{x})\right]$. This is true at once for all possible decompositions of the signal into components and irrespective of what particular linear operator is used. The key to understanding this is to keep in mind that the component modulating functions become uniquely determined only after an imaginary part is added to the signal and a decomposition into components is specified. For any given decomposition of $s(\boldsymbol{x})$ into components, different linear operators will compute different imaginary parts for the specified components and will therefore lead to different solutions for the component modulating functions.
Let $\boldsymbol{e}_{i}$ be the unit vector in an arbitrarily chosen direction $x_{i}$. The method we favor for generating the complex extension is to add an imaginary part equal to $j$ times the directional multidimensional Hilbert transform [21]-[23]

$$
\begin{aligned}
\mathcal{H}[s(\boldsymbol{x})] & =\text { p.v. } \frac{1}{\pi} \int_{\mathbb{R}} s\left(\boldsymbol{x}-\xi \boldsymbol{e}_{i}\right) \frac{d \xi}{\xi} \\
& =s(\boldsymbol{x}) * \frac{1}{\pi \boldsymbol{x}^{T} \boldsymbol{e}_{i}} \prod_{k \neq i} \delta\left(\boldsymbol{x}^{T} \boldsymbol{e}_{k}\right)
\end{aligned}
$$

where p.v. indicates the Cauchy principal value. It is trivial to verify that the singular operator $\mathcal{H}$ is linear. The complex signal $t(\boldsymbol{x})=s(\boldsymbol{x})+j \mathcal{H}[s(\boldsymbol{x})]$ admits many of the attractive properties of the 1-D analytic signal. ${ }^{1}$ Thus, by abus de langage, we call $t(\boldsymbol{x})$ the analytic image associated with the real signal $s(\boldsymbol{x})$. Over the Hilbert space $L^{2}\left(\mathbb{R}^{n}\right), t(x)$ satisfies the frequency moment properties of Gabor and Ville [24], [25], the amplitude continuity, homogeneity, and harmonic correspondence conditions of Vakman [26] (up to a set of Lebesgue measure zero), and admits a Fourier spectrum that is supported only on $n / 2$ frequency orthants where it is equal to twice the spectrum of $s(\boldsymbol{x})$. Although it is not difficult to extend the discrete Hilbert transform given in [27] into multiple dimensions to discretize (7), the details are beyond the scope of this paper.

The complex extension $t(\boldsymbol{x})$ and associated transform (7) are distinct from the ones used for 2-D Wigner analysis by Zhu et al. [28], the 2-D discrete Hilbert transform used by Read and Treitel for stabilizing IIR filters [29], and Hahn's complex signals with single-orthant spectra [30]. Indeed, none of these admit all of the attractive properties mentioned above. Therefore, for computing multidimensional AM-FM models, we feel that the analytic image is the preferable complex extension. Accordingly, for any given component-wise decomposition, we define the component modulating functions of the real signal $s(x)$ to be those of its associated analytic image $t(\boldsymbol{x})=s(\boldsymbol{x})+j \mathcal{H}[s(\boldsymbol{x})]$.

Multiband linear filtering to isolate the multiple signal components is a crucial step in the DCA and CCA paradigms. If this linear filtering is implemented via pointwise spectral multiplication, as it typically is for discrete multidimensional signals, then generation of the analytic image can be incorporated directly into the filterbank by zeroing half of each filter's spectrum. Thus there is no additional computational overhead in computing the extension. In fact, the number of multiplications required to compute each filter response is reduced by half.

\section{Multidimensional QuASI-EIGENFUnCTION APPROXIMATIONS AND DEMODULATION ALGORITHMS}

Analysis of the behavior of AM-FM signals in LSI systems is difficult because a general closed-form expression for the system response cannot be obtained in terms of the input modulating functions. In this section, we introduce new approximations for the responses of $n \mathrm{D}$ LSI systems to AM-FM inputs and develop bounds on the approximation errors. We use the approximations to develop novel $n \mathrm{D}$ algorithms for demodulating a signal directly and also for estimating the signal's modulating functions from the response of an LSI filter. It will be convenient

\footnotetext{
${ }^{1}$ In general, however, it may fail to satisfy the multidimensional Cauchy-Riemann equations.
} 
to restrict our attention to single component AM-FM signals up until Section III-E.

Let $g: \mathbb{R}^{n} \rightarrow \mathbb{C}$ be the impulse response of an LSI system with frequency response $G(\boldsymbol{\Omega})$. If the system input is $t(\boldsymbol{x})=$ $A_{0} \exp \left[j \Omega_{0}^{T} x\right]$, where $A_{0}$ and $\Omega_{0}$ are fixed, then the response is given exactly by

$$
y(\boldsymbol{x})=t(\boldsymbol{x}) * g(\boldsymbol{x})=t(\boldsymbol{x}) G\left(\boldsymbol{\Omega}_{0}\right) .
$$

In this case, the monochromatic input $t(x)$ is an eigenfunction of the system and the complex number $G\left(\boldsymbol{\Omega}_{0}\right)$ is the associated eigenvalue. For a general AM-FM input $t(\boldsymbol{x})=a(\boldsymbol{x}) e^{j \varphi(\boldsymbol{x})},(8)$ motivates the QEA

$$
y(\boldsymbol{x}) \approx \hat{y}(\boldsymbol{x})=t(\boldsymbol{x}) G[\nabla \varphi(\boldsymbol{x})]
$$

Whereas the eigenvalue $G\left(\boldsymbol{\Omega}_{0}\right)$ in (8) is a constant, the term $G[\nabla \varphi(x)]$ in (9) varies with $\boldsymbol{x}$. Over neighborhoods where the approximation holds well, this term locally characterizes the transmission of $t(x)$ through the system.

When the input modulating functions are sufficiently smooth in a region that is sufficiently large [with relation to the significant support of $g(\boldsymbol{x})$ ], accuracy of the QEA (9) is generally excellent. This notion is made rigorous in Sections III-A and III-C, where we bound the approximation error by the effective duration (i.e., spatial concentration) of $g(x)$ and by the smoothness functionals $\overline{\mathcal{D}^{q}}(a), \mathfrak{L}^{1}(a), \overline{\mathcal{S}^{q}}(\varphi)$, and $\mathfrak{S}_{i}^{1}(\varphi)$. QEA's are most useful, in the sense of incurring small errors, when applied to signals that admit a decomposition (1) wherein each component might be globally wideband, but is locally narrowband over much of the domain. On a global scale, such components may bear little resemblance to true eigenfunctions. In the examples of Section V, we demonstrate that this characterization is applicable to 2-D images of general practical interest.

In the remainder of the paper, it will at times be desirable to write a vector in terms of its components using a compact, nonstandard notation. We will use angle brackets for this purpose. Thus, we will write $\boldsymbol{x}=\left[\begin{array}{llll}x_{1} & x_{2} & \cdots & x_{n}\end{array}\right]^{T}=\left\langle x_{i}\right\rangle$, where it is understood that the index $i$ runs from 1 to $n$. We will also write expressions such as $\left\langle\boldsymbol{p}^{T} \nabla \varphi_{x_{i}}(\boldsymbol{p})\right\rangle, \boldsymbol{p} \in \mathbb{Z}^{n}$, which are interpreted as follows. For $i \in[1, n], \varphi_{x_{i}}(\boldsymbol{p})$ are the samples of the partials of $\varphi$. The gradient $\nabla \varphi_{x_{i}}$ of each partial is a vector, and, for each $i, \boldsymbol{p}^{T} \nabla \varphi_{x_{i}}(\boldsymbol{p})$ takes scalar values. The vector with these scalars as entries is denoted $\left\langle\boldsymbol{p}^{T} \nabla \varphi_{x_{i}}(\boldsymbol{p})\right\rangle$.

\section{A. Continuous QEA's and Error Bounds}

For the signal $t(\boldsymbol{x})=a(\boldsymbol{x}) e^{j \varphi(\boldsymbol{x})}$, let $a_{\max }=\sup _{\boldsymbol{x} \in \mathbb{R}^{n}} a(\boldsymbol{x})$ and denote the absolute error in the QEA (9) by $\varepsilon_{y}(\boldsymbol{x})=\mid y(\boldsymbol{x})-$ $\hat{y}(\boldsymbol{x}) \mid$. Theorem 1 below places a uniform upper bound on $\varepsilon_{y}(\boldsymbol{x})$. The functionals $\overline{\boldsymbol{\Delta}^{p}}(g)$ and $\overline{\boldsymbol{\Theta}^{p}}(g)$ are defined after the theorem is stated.

Theorem 1: Let $n$ be finite and let $p$ and $q$ be any pair of conjugate exponents, $p q=p+q$, such that $q>n$. Then

$$
\begin{aligned}
\varepsilon_{y}(\boldsymbol{x}) \leq & \frac{q}{q-n} \overline{\boldsymbol{\Delta}^{p}}(g) \overline{\mathcal{D}^{q}}(a) \\
& +\frac{q^{2} a_{\max }}{(q-n)(2 q-n)} \overline{\boldsymbol{\Theta}^{p}}(g) \overline{\mathcal{S}^{q}}(\varphi) .
\end{aligned}
$$

Proof: The lengthy proof, which is omitted for brevity, may be found in [31] and proceeds along arguments similar to those used to prove [11, Theorem 1]. The dimensionally dependent terms $q /(q-n)$ and $q^{2} a_{\max } /\{(q-n)(2 q-n)\}$ emerge as natural conditions for convergence of the involved integrals.

The bound (10) fails to converge unless $q>n$, which implies $p<n /(n+1)$. In the limit as $q \rightarrow \infty$ and $p \rightarrow 1$, the terms $q /(q-n)$ and $q^{2} a_{\max } /\{(q-n)(2 q-n)\}$ approach one and $a_{\max } / 2$, respectively. In Theorem 1 , the spatial concentration of $g(\boldsymbol{x})$ is quantified in terms of generalized directional $p$-energy variances

$$
\boldsymbol{\Delta}_{i}^{p}(g)=\left[\int_{\mathbb{R}^{n}}\left|x_{i} g(\boldsymbol{x})\right|^{p} d \boldsymbol{x}\right]^{1 / p}
$$

and cross-variances

$$
\boldsymbol{\Theta}_{i, j}^{p}(g)=\left[\int_{\mathbb{R}^{n}}\left|x_{i} x_{j} g(\boldsymbol{x})\right|^{p} d \boldsymbol{x}\right]^{1 / p}
$$

where $1 \leq i, j \leq n$. These functionals grow as the effective spatial support of the filter increases, while they vanish in the limit as the filter support is reduced to a point. The functional $\overline{\Delta^{p}}(g)$ in (10), which simultaneously quantifies the concentration of the filter in all directions, is the $\ell^{p}$-norm of the sequence $\left\{\boldsymbol{\Delta}_{i}^{p}(g)\right\}, i \in[1, n]$. Similarly, $\overline{\boldsymbol{\Theta}^{p}}(g)=\left\|\Theta_{i, j}^{p}(g)\right\|_{\ell^{p}}$.

The error bound of Theorem 1 is tight in the sense that $\overline{\mathcal{D}^{q}}(a)$ and $\overline{\mathcal{S}^{q}}(\varphi)$ both go to zero in the limit as $t(\boldsymbol{x})$ becomes monochromatic, verifying that there is no error in (9) when the input is a true eigenfunction. The bound is a useful analytical tool because the individual contributions of each modulating function and of the filter impulse response are clearly articulated. Note that the bound can be made uniformly small independent of the modulating functions by designing $g(x)$ to minimize $\overline{\boldsymbol{\Delta}^{p}}(g)$ and $\overline{\boldsymbol{\Theta}^{p}}(g)$. When $n=1$ or 2, (10) collapses to the bounds given previously in [3] and [11].

In Section III-B, we will develop continuous demodulation algorithms and the need will arise to apply a QEA when the system input is the partial derivative

$$
t_{x_{i}}(\boldsymbol{x})=a_{x_{i}}(\boldsymbol{x}) \exp [j \varphi(\boldsymbol{x})]+j a(\boldsymbol{x}) \varphi_{x_{i}}(\boldsymbol{x}) \exp [j \varphi(\boldsymbol{x})]
$$

$$
=\left[a_{x_{i}}(\boldsymbol{x})+j a(\boldsymbol{x}) \varphi_{x_{i}}(\boldsymbol{x})\right] \exp [j \varphi(\boldsymbol{x})]
$$

where $t(\boldsymbol{x})=a(\boldsymbol{x}) e^{j \varphi(\boldsymbol{x})}$ as before. Let

$$
\psi_{i}(\boldsymbol{x})=t_{x_{i}}(\boldsymbol{x}) * g(\boldsymbol{x})
$$

and define the QEA

$$
\psi_{i}(\boldsymbol{x}) \approx \hat{\psi}_{i}(\boldsymbol{x})=t_{x_{i}}(\boldsymbol{x}) G[\nabla \varphi(\boldsymbol{x})] .
$$

The absolute error in this approximation is $\varepsilon_{\psi_{i}}(\boldsymbol{x})=$ $\left|\psi_{i}(\boldsymbol{x})-\hat{\psi}_{i}(\boldsymbol{x})\right|$. Note that the approximations (16) and (9) are different: the AM functions $a_{x_{i}}(\boldsymbol{x})$ and $a(\boldsymbol{x}) \varphi_{x_{i}}(\boldsymbol{x})$ in (15) might not be positive semidefinite. Thus, Theorem 1 cannot be used directly to establish a bound on $\varepsilon_{\psi_{i}}(\boldsymbol{x})$. Approximations such as (16) have not been considered before. Let $a^{+}(\boldsymbol{x})=\max \{a(\boldsymbol{x}), 0\}$ and $a^{-}(\boldsymbol{x})=-\min \{a(\boldsymbol{x}), 0\}$. Clearly, $a(\boldsymbol{x})=a^{+}(\boldsymbol{x})-a^{-}(\boldsymbol{x})$. Moreover, $a^{+}(\boldsymbol{x})$ and $a^{-}(\boldsymbol{x})$ 
are both positive semidefinite. With this notation, (13) may be written as

$$
\begin{aligned}
t_{x_{i}}(\boldsymbol{x})= & a_{x_{i}}^{+}(\boldsymbol{x}) \exp [j \varphi(\boldsymbol{x})]-a_{x_{i}}^{-}(\boldsymbol{x}) \exp [j \varphi(\boldsymbol{x})] \\
& +j a(\boldsymbol{x}) \varphi_{x_{i}}^{+}(\boldsymbol{x}) \exp [j \varphi(\boldsymbol{x})] \\
& -j a(\boldsymbol{x}) \varphi_{x_{i}}^{-}(\boldsymbol{x}) \exp [j \varphi(\boldsymbol{x})] .
\end{aligned}
$$

We now give a corollary to Theorem 1 which uniformly bounds $\varepsilon_{\psi_{i}}(\boldsymbol{x})$. The following definition is used in the hypothesis.

Definition: A point $\boldsymbol{x}_{0} \in \mathbb{R}^{n}$ is a zero crossing of the function $a(\boldsymbol{x})$ if every open neighborhood about $\boldsymbol{x}_{0}$ contains a point $\boldsymbol{x}^{+}$such that $\operatorname{sgn}\left[a\left(\boldsymbol{x}^{+}\right)\right]=1$ and also contains a point $\boldsymbol{x}^{-}$such that $\operatorname{sgn}\left[a\left(\boldsymbol{x}^{-}\right)\right]=-1$. The set of all such points $\boldsymbol{x}_{0}$ is the zero crossing set of $a(\boldsymbol{x})$.

Corollary 1: Let $n$ be finite and let $p$ and $q$ be conjugate exponents with $q>n$. Let $a(\boldsymbol{x})$ and $\varphi(\boldsymbol{x})$ be such that the zero crossing sets of $a_{x_{i}}(\boldsymbol{x})$ and $\varphi_{x_{i}}(\boldsymbol{x})$ have Lebesgue measure zero. Then

$$
\begin{aligned}
\varepsilon_{\psi_{i}}(\boldsymbol{x}) \leq & \frac{q}{q-n} \overline{\boldsymbol{\Delta}^{p}}(g)\left\{\overline{\mathcal{D}^{q}}\left(a_{x_{i}}^{+}\right)+\overline{\mathcal{D}^{q}}\left(a_{x_{i}}^{-}\right)+\overline{\mathcal{D}^{q}}\left(a \varphi_{x_{i}}^{+}\right)\right. \\
& \left.+\overline{\mathcal{D}^{q}}\left(a \varphi_{x_{i}}^{-}\right)\right\}+\frac{q^{2} a_{\max }}{(q-n)(2 q-n)} \overline{\boldsymbol{\Theta}^{p}}(g) \overline{\mathcal{S}^{q}}(\varphi) .
\end{aligned}
$$

Proof: See the Appendix.

The assumption that $a_{x_{i}}(\boldsymbol{x})$ and $\varphi_{x_{i}}(\boldsymbol{x})$ admit zero crossing sets of Lebesgue measure zero is a mild one. It can fail only if the partials of the modulating functions cross zero uncountably many times, a condition that is not expected to occur in signals of practical interest. Interpretation of (18) is similar to that of (10). The bound falls to zero in the limit as $t_{x_{i}}(\boldsymbol{x})$ tends toward a true eigenfunction, which implies that $t(\boldsymbol{x})$ also tends toward a true eigenfunction. As before, the errors in (16) can be made uniformly small by designing $g(\boldsymbol{x})$ to minimize $\overline{\boldsymbol{\Delta}^{p}}(g)$ and $\overline{\boldsymbol{\Theta}^{p}}(g)$. For signals that are reasonably locally narrowband, errors in the QEA's (9) and (16) may be expected to be small or negligible.

\section{B. Continuous Demodulation}

For any arbitrary single component AM-FM signal $t(\boldsymbol{x})=$ $a(\boldsymbol{x}) e^{j \varphi(\boldsymbol{x})}$, one may verify by direct calculation that, at points where $t(x) \neq 0$, the demodulation algorithms

$$
\begin{gathered}
\nabla \varphi(\boldsymbol{x})=\operatorname{Re}\left[\frac{\nabla t(\boldsymbol{x})}{j t(\boldsymbol{x})}\right] \\
a(\boldsymbol{x})=|t(\boldsymbol{x})|
\end{gathered}
$$

are exact. ${ }^{2}$ In DCA and CCA, however, the modulating functions must be estimated from the channel responses of a multiband linear filterbank. In this section, we derive filtered demodulation algorithms for this purpose. Let $\psi(\boldsymbol{x})=\left\langle\psi_{i}(\boldsymbol{x})\right\rangle$ and $\hat{\psi}(\boldsymbol{x})=$ $\left\langle\hat{\psi}_{i}(\boldsymbol{x})\right\rangle$, where $\psi_{i}(\boldsymbol{x})$ and $\hat{\psi}_{i}(\boldsymbol{x})$ were defined in (15) and (16). Let $g(\boldsymbol{x})$ be the impulse response of an LSI filter and let $y(\boldsymbol{x})=$

${ }^{2}$ At points where $t(\boldsymbol{x})=0,(20)$ indicates that $a(\boldsymbol{x})=0$. Thus, the instantaneous values of $\varphi(\boldsymbol{x})$ and $\nabla \varphi(\boldsymbol{x})$ at such points are immaterial to the equality in (1). $t(\boldsymbol{x}) * g(\boldsymbol{x})$, where $t(\boldsymbol{x})=a(\boldsymbol{x}) e^{j \varphi(\boldsymbol{x})}$. Since differentiation and convolution commute, we have that

$$
\nabla y(\boldsymbol{x})=g(\boldsymbol{x}) * \nabla t(\boldsymbol{x})=\psi(\boldsymbol{x}) .
$$

Written in vector form, the QEA (16) then becomes

$$
\psi(\boldsymbol{x}) \approx \hat{\psi}(\boldsymbol{x})=\nabla t(\boldsymbol{x}) G[\nabla \varphi(\boldsymbol{x})] .
$$

Upon applying the frequency demodulation algorithm (19) directly to $y(\boldsymbol{x})$ and subsequently applying (22) to the numerator and (9) to the denominator, we obtain

$$
\begin{aligned}
\operatorname{Re}\left[\frac{\nabla y(\boldsymbol{x})}{j y(\boldsymbol{x})}\right] & =\operatorname{Re}\left[\frac{\psi(\boldsymbol{x})}{j y(\boldsymbol{x})}\right] \approx \operatorname{Re}\left[\frac{\hat{\psi}(\boldsymbol{x})}{j \hat{y}(\boldsymbol{x})}\right] \\
& =\operatorname{Re}\left[\frac{\nabla t(\boldsymbol{x})}{j t(\boldsymbol{x})}\right] .
\end{aligned}
$$

The approximation error in each component of the numerator of (23) must everywhere fall below the bound of Corollary 1 , while that in the denominator must everywhere fall below the bound of Theorem 1. Comparison of (24) with (19) validates the filtered frequency demodulation algorithm

$$
\nabla \varphi(\boldsymbol{x}) \approx \nabla \hat{\varphi}(\boldsymbol{x})=\operatorname{Re}\left[\frac{\nabla y(\boldsymbol{x})}{j y(\boldsymbol{x})}\right] .
$$

This important result shows that, at points where $y(x) \neq 0$, the FM function of a locally narrowband signal may be estimated directly from the filter response. Note that, in a practical implementation, $\nabla \varphi(\boldsymbol{x})$ can be estimated at points where $y(\boldsymbol{x}) \approx 0$ by interpolating the values of $\nabla \hat{\varphi}(\boldsymbol{x})$ at nearby points where $y(x) \neq 0$.

To estimate the AM function of $t(\boldsymbol{x})$, we apply (9) on top and bottom to obtain

$$
\left|\frac{y(\boldsymbol{x})}{G[\nabla \hat{\varphi}(\boldsymbol{x})]}\right| \approx\left|\frac{t(\boldsymbol{x}) G[\nabla \varphi(\boldsymbol{x})]}{G[\nabla \varphi(\boldsymbol{x})]}\right|=a(\boldsymbol{x}) .
$$

This motivates the filtered amplitude demodulation algorithm

$$
a(\boldsymbol{x}) \approx \hat{a}(\boldsymbol{x})=\left|\frac{y(\boldsymbol{x})}{G[\nabla \hat{\varphi}(\boldsymbol{x})]}\right|,
$$

which may be applied to estimate $a(\boldsymbol{x})$ from $y(\boldsymbol{x})$ once $\nabla \hat{\varphi}(\boldsymbol{x})$ has been calculated using (25).

\section{Discrete QEA and Error Bounds}

In this section, we present a new multidimensional discrete QEA analogous to (9) and develop bounds on the approximation error. Let $t(\boldsymbol{x})=a(\boldsymbol{x}) e^{j \varphi(\boldsymbol{x})}$ as before, and let $t(\boldsymbol{k}): \mathbb{Z}^{n} \rightarrow \mathbb{C}$ contain the samples of $t(\boldsymbol{x})$. Let $a_{\max }=\sup _{\boldsymbol{k} \in \mathbb{Z}^{n}} a(\boldsymbol{k})$ and suppose that $g(\boldsymbol{k}) \in \ell^{1}\left(\mathbb{Z}^{n}\right)$ is the unit pulse response of a discrete LSI system with frequency response $G(\boldsymbol{\omega})$. The system response $y(\boldsymbol{k})=t(\boldsymbol{k}) * g(\boldsymbol{k})$ is given exactly by

$$
y(\boldsymbol{k})=\sum_{\boldsymbol{p} \in \mathbb{Z}^{n}} g(\boldsymbol{p}) t(\boldsymbol{k}-\boldsymbol{p}) .
$$

In analogy to (9), the QEA for the response is

$$
\hat{y}(\boldsymbol{k})=t(\boldsymbol{k}) G[\nabla \varphi(\boldsymbol{k})],
$$


while the absolute approximation error is $\varepsilon_{y}(\boldsymbol{k})=|y(\boldsymbol{k})-\hat{y}(\boldsymbol{k})|$. The following theorem locally bounds $\varepsilon_{y}(\boldsymbol{k})$.

Theorem 2: Let $n$ be finite and let $g(k) \in \ell^{1}\left(\mathbb{Z}^{n}\right)$. Then

$$
\begin{aligned}
\varepsilon_{y}(\boldsymbol{k}) \leq & \sum_{\substack{\boldsymbol{p} \mathbb{Z}^{n} \\
\boldsymbol{p} \neq \mathbf{0}}}|g(\boldsymbol{p})|\{|a(\boldsymbol{k}-\boldsymbol{p})-a(\boldsymbol{k})| \\
& \left.+a_{\max } \int_{0}^{1}\left|\boldsymbol{p}^{T}\left\langle\boldsymbol{p}^{T} \nabla \varphi_{x_{i}}(k-s \boldsymbol{p})\right\rangle\right| d s\right\} .
\end{aligned}
$$

Proof: See the Appendix.

In (30), the absolute approximation error incurred by (29) is bounded by a path integral quantifying the smoothness of $\nabla \varphi(\boldsymbol{x})$ and by the absolute deviations of $a(\boldsymbol{k})$, both weighted by the magnitudes of the filter coefficients. For filters that are spatially localized, only the local behavior of the modulating functions contributes significantly to the bound. For locally narrowband signals, the local amplitude deviations and second partials of $\varphi(x)$ are both expected to have small magnitudes. Theorem 2 is of practical interest because (30) is spatially varying and locally tracks the QEA error. The bound can be studied numerically for any particular combination of signal and filter. We next introduce a corollary to Theorem 2 which bounds $\varepsilon_{y}(\boldsymbol{k})$ uniformly.

Corollary 2: Assume the hypothesis of Theorem 2. Then

$$
\varepsilon_{y}(\boldsymbol{k}) \leq\left\{\|g\|_{\ell^{1}}-|g(\mathbf{0})|\right\} \mathfrak{L}^{1}(a)+a_{\max }\left\langle\mathfrak{A}_{i}(g)\right\rangle^{T}\left\langle\mathfrak{S}_{i}^{1}(\varphi)\right\rangle .
$$

Proof: See the Appendix.

As with Theorem 1 and Corollary 1, the individual contributions of each modulating function and of $g(k)$ are clearly articulated in the bound of Corollary 2. Dependence of the bound on the spatial concentration of $g(\boldsymbol{k})$ is through the generalized directional energy moment functional

$$
\mathfrak{A}_{i}(g)=\sum_{\boldsymbol{k} \in \mathbb{Z}^{n}}\left|\boldsymbol{k} \boldsymbol{e}_{i}^{T} \boldsymbol{k}\right||g(\boldsymbol{k})|
$$

and through the $\ell^{1}$-norm modified by deletion of $|g(\mathbf{0})|$. This has the interesting consequence that both (30) and (31) are independent of $g(\mathbf{0})$. If the filter unit pulse response is the Kronecker delta, then both bounds are identically zero, reflecting the fact that all inputs are then true eigenfunctions. The local bound of Theorem 2 is always equal to or below the uniform bound of Corollary 2. Both bounds are tight in the sense that they vanish in the limit as $t(k)$ becomes monochromatic.

\section{Discrete Demodulation}

In this section, we use the QEA of Section III-C to develop new amplitude and frequency demodulation algorithms applicable to an $n \mathrm{D}$ discrete AM-FM signal $t(\boldsymbol{k})=a(\boldsymbol{k}) e^{j \varphi(\boldsymbol{k})}$. Clearly, $a(\boldsymbol{k})=|t(\boldsymbol{k})|$. However, an exact algorithm analogous to (19) does not exist in the discrete case. Thus, even for an unfiltered signal, some labor is required to perform frequency demodulation. Consider a discrete LSI system with unit pulse response

$$
h_{i}(\boldsymbol{k})=\delta\left(\boldsymbol{k}+n_{1} \boldsymbol{e}_{i}\right)+q \delta\left(\boldsymbol{k}+n_{2} \boldsymbol{e}_{i}\right)
$$

where $\delta(\boldsymbol{k})$ is the Kronecker delta and constants $n_{1}, n_{2} \in \mathbb{Z}$ and $q \in\{-1,+1\}$ are parameters. The system frequency response is $H_{i}(\boldsymbol{\omega})=e^{j n_{1} \boldsymbol{\omega}^{T} \boldsymbol{e}_{i}}+q e^{j n_{2} \boldsymbol{\omega}^{T} \boldsymbol{e}_{i}}$. The system output is given exactly by

$$
y(k)=t(\boldsymbol{k}) * h_{i}(\boldsymbol{k})=t\left(\boldsymbol{k}+n_{1} \boldsymbol{e}_{i}\right)+q t\left(\boldsymbol{k}+n_{2} \boldsymbol{e}_{i}\right) .
$$

Applying the QEA (29), we obtain

$$
\hat{y}(\boldsymbol{k})=t(\boldsymbol{k})\left\{\exp \left[j n_{1} \boldsymbol{e}_{i}^{T} \nabla \varphi(\boldsymbol{k})\right]+q \exp \left[j n_{2} \boldsymbol{e}_{i}^{T} \nabla \varphi(\boldsymbol{k})\right]\right\} .
$$

Taking $n_{1}=+1$ and $n_{2}=q=-1$, equating the right sides of (34) and (35) to within approximation errors, and subsequently dividing through by $t(k)$ and applying a routine series of trigonometric substitutions leads almost immediately to the spatially localized discrete frequency algorithm

$$
\boldsymbol{e}_{i}^{T} \nabla \varphi(\boldsymbol{k}) \approx \boldsymbol{e}_{i}^{T} \nabla \hat{\varphi}(\boldsymbol{k})=\arcsin \left[\frac{t\left(\boldsymbol{k}+\boldsymbol{e}_{i}\right)-t\left(\boldsymbol{k}-\boldsymbol{e}_{i}\right)}{2 j t(\boldsymbol{k})}\right]
$$

applicable at points where $t(\boldsymbol{k}) \neq 0$. Alternatively, taking $n_{1}=$ $q=+1$ and $n_{2}=-1$ gives

$$
\boldsymbol{e}_{i}^{T} \nabla \varphi(\boldsymbol{k}) \approx \boldsymbol{e}_{i}^{T} \nabla \hat{\varphi}(\boldsymbol{k})=\arccos \left[\frac{t\left(\boldsymbol{k}+\boldsymbol{e}_{i}\right)+t\left(\boldsymbol{k}-\boldsymbol{e}_{i}\right)}{2 t(\boldsymbol{k})}\right] .
$$

To within QEA errors and numerical roundoff errors, the arguments of the transcendentals in (36) and (37) will have imaginary components that are equal to zero. In a practical implementation, any nonzero imaginary component should be discarded before the transcendentals are evaluated. Because arcsin and arccos are multivalued, the frequency estimates delivered individually by (36) and (37) are ambiguous by an additive factor of $k \pi, k \in \mathbb{Z}$. However, the algorithms can be used together to correctly place the estimated frequencies in the interval $[-\pi, \pi]$.

In view of the fact that DCA and CCA must estimate the modulating functions of $t(\boldsymbol{k})$ from filterbank channel responses, we next develop discrete filtered demodulation algorithms analogous to (25) and (27). Let $g(\boldsymbol{k})$ and $G(\boldsymbol{\omega})$ be the unit pulse response and frequency response of an LSI filter, where $g(\boldsymbol{k}) \in$ $\ell^{1}\left(\mathbb{Z}^{n}\right)$. The system output $y(\boldsymbol{k})=t(\boldsymbol{k}) * g(\boldsymbol{k})$ is given by (28). As in the continuous case, we will establish the validity of applying the unfiltered frequency algorithms (36) and (37) directly to $y(\boldsymbol{k})$. Consider a system formed by cascading (33) with $g(\boldsymbol{k})$. The unit pulse response of the cascade system is $h_{i}(\boldsymbol{k}) * g(\boldsymbol{k})=g\left(\boldsymbol{k}+n_{1} \boldsymbol{e}_{i}\right)+q g\left(\boldsymbol{k}+n_{2} \boldsymbol{e}_{i}\right)$, while the frequency response is $G(\boldsymbol{\omega})\left[e^{j n_{1} \boldsymbol{\omega}^{T} \boldsymbol{e}_{i}}+q e^{j n_{2} \boldsymbol{\omega}^{T} \boldsymbol{e}_{i}}\right]$. Let the response of the cascade system be $\xi(\boldsymbol{k})=t(\boldsymbol{k}) * g(\boldsymbol{k}) * h_{i}(\boldsymbol{k})=y(\boldsymbol{k}) * h_{i}(\boldsymbol{k})$. Then $\xi(\boldsymbol{k})$ may be written in terms of $y(\boldsymbol{k})$ according to

$$
\xi(\boldsymbol{k})=\sum_{\boldsymbol{p} \in \mathbb{Z}^{n}} h_{i}(\boldsymbol{p}) y(\boldsymbol{k}-\boldsymbol{p})=y\left(\boldsymbol{k}+n_{1} \boldsymbol{e}_{i}\right)+q y\left(\boldsymbol{k}+n_{2} \boldsymbol{e}_{i}\right) .
$$

Applying the QEA (29) to the overall cascade system yields

$$
\begin{aligned}
\hat{\xi}(\boldsymbol{k})= & t(\boldsymbol{k}) G[\nabla \varphi(\boldsymbol{k})]\left\{\exp \left[j n_{1} \boldsymbol{e}_{i}^{T} \nabla \varphi(\boldsymbol{k})\right]\right. \\
& \left.+q \exp \left[j n_{2} \boldsymbol{e}_{i}^{T} \nabla \varphi(\boldsymbol{k})\right]\right\} .
\end{aligned}
$$


Now, the approximation (29) may also be applied to the system $g(\boldsymbol{k})$ alone to obtain $t(\boldsymbol{k}) G[\nabla \varphi(\boldsymbol{k})]=\hat{y}(\boldsymbol{k}) \approx y(\boldsymbol{k})$. Substituting this result into (39) gives

$$
\hat{\xi}(\boldsymbol{k}) \approx y(\boldsymbol{k})\left\{\exp \left[j n_{1} \boldsymbol{e}_{i}^{T} \nabla \varphi(\boldsymbol{k})\right]+q \exp \left[j n_{2} \boldsymbol{e}_{i}^{T} \nabla \varphi(\boldsymbol{k})\right]\right\}
$$

Upon equating the right sides of (38) and (40), dividing through by $y(\boldsymbol{k})$, and taking $n_{1}=+1$ and $n_{2}=q=-1$, we obtain

$$
\boldsymbol{e}_{i}^{T} \nabla \varphi(\boldsymbol{k}) \approx \boldsymbol{e}_{i}^{T} \nabla \hat{\varphi}(\boldsymbol{k})=\arcsin \left[\frac{y\left(\boldsymbol{k}+\boldsymbol{e}_{i}\right)-y\left(\boldsymbol{k}-\boldsymbol{e}_{i}\right)}{2 j y(\boldsymbol{k})}\right] .
$$

Likewise, choosing $n_{1}=q=+1$ and $n_{2}=-1$ yields

$$
\boldsymbol{e}_{i}^{T} \nabla \varphi(\boldsymbol{k}) \approx \boldsymbol{e}_{i}^{T} \nabla \hat{\varphi}(\boldsymbol{k})=\arccos \left[\frac{y\left(\boldsymbol{k}+\boldsymbol{e}_{i}\right)+y\left(\boldsymbol{k}-\boldsymbol{e}_{i}\right)}{2 y(\boldsymbol{k})}\right] \text {. }
$$

Thus, each component of $\nabla \varphi(\boldsymbol{k})$ may be estimated from $y(\boldsymbol{k})$ at points where $y(\boldsymbol{k}) \neq 0$. Once $\nabla \hat{\varphi}(\boldsymbol{k})$ has been obtained, a final application of the QEA (29) validates the filtered amplitude demodulation algorithm

$$
a(\boldsymbol{k}) \approx \hat{a}(\boldsymbol{k})=\left|\frac{y(\boldsymbol{k})}{G[\nabla \hat{\varphi}(\boldsymbol{k})]}\right| .
$$

\section{E. Component Demodulation}

We now return our attention to the multicomponent model (1) and the DCA and CCA computational paradigms. For both techniques, the structure of the filterbank plays an important role in determining the multicomponent interpretation of the signal. With CCA the component-wise decomposition is carried out by assuming that each channel response is globally dominated by a single component, whereas DCA assumes that at most one component dominates each response at each point. For the continuous-domain case, suppose that $g_{m}(\boldsymbol{x})$ and $G_{m}(\boldsymbol{\Omega})$ are the impulse response and frequency response of filterbank channel $m$, and let $y_{m}(\boldsymbol{x})=t(\boldsymbol{x}) * g_{m}(\boldsymbol{x})$ be the channel response. Suppose further that component $t_{i}(\boldsymbol{x})$ dominates $y_{m}(\boldsymbol{x})$ at the point $x_{0}$, so that $\left.y_{m}\left(x_{0}\right) \approx t_{i}(\boldsymbol{x}) * g_{m}(\boldsymbol{x})\right|_{x=x_{0}}$. Since the frequency algorithm (25) operates on a pointwise basis, $\nabla \varphi_{i}(\boldsymbol{x})$ may be estimated at the point $\boldsymbol{x}_{0}$ by

$$
\nabla \varphi_{i}\left(\boldsymbol{x}_{0}\right) \approx \nabla \hat{\varphi}_{i}\left(\boldsymbol{x}_{0}\right)=\operatorname{Re}\left[\nabla y_{m}(\boldsymbol{x}) /\left.\left\{j y_{m}(\boldsymbol{x})\right\}\right|_{\boldsymbol{x}=\boldsymbol{x}_{0}} .\right.
$$

Subsequently, $a_{i}(\boldsymbol{x})$ may be estimated at $\boldsymbol{x}_{0}$ by applying (27) to the response of channel $m$ to obtain

$$
a_{i}\left(\boldsymbol{x}_{0}\right) \approx \hat{a}_{i}\left(\boldsymbol{x}_{0}\right)=\left|y_{m}(\boldsymbol{x}) / G_{m}\left[\nabla \hat{\varphi}_{i}(\boldsymbol{x})\right]\right|_{\boldsymbol{x}=\boldsymbol{x}_{0}} .
$$

Since the algorithms (41)-(43) are also highly spatially localized, an identical line of reasoning may be applied in the discrete case.

\section{DCA AND CCA COMPUTATIONAL TECHNIQUES}

\section{A. DCA}

A block diagram of DCA appears in Fig. 1, where $t(\boldsymbol{x})$ is analyzed with an $M$-channel filterbank to isolate components on a jointly localized basis. The demodulation algorithms (25) and (27) [or (41)-(43) in the discrete case] are applied in the blocks marked DEMOD. We define the dominant component at each point to be the one that dominates the response of the channel that maximizes the channel selection criterion

$$
\Psi_{m}(\boldsymbol{x})=\frac{\left|y_{m}(\boldsymbol{x})\right|}{\max _{\boldsymbol{\Omega}}\left|G_{m}(\boldsymbol{\Omega})\right|}
$$

and we extract estimates of the dominant modulations $a_{D}(\boldsymbol{x})$ and $\nabla \varphi_{D}(\boldsymbol{x})$ from the maximizing channel on a pointwise basis. Use of the criterion (44) is motivated by the assumption that some particular component $t_{i}(\boldsymbol{x})$ dominates the channel response at any given point $x$, so that $y_{m}(\boldsymbol{x}) \approx t_{i}(\boldsymbol{x}) * g_{m}(\boldsymbol{x})$ at the point. Upon application of the QEA $y_{m}(\boldsymbol{x}) \approx t_{i}(\boldsymbol{x}) G_{m}\left[\nabla \varphi_{i}(\boldsymbol{x})\right]$, (44) becomes

$$
\Psi_{m}(\boldsymbol{x}) \approx\left|a_{i}(\boldsymbol{x})\right| \frac{\left|G_{m}\left[\nabla \varphi_{i}(\boldsymbol{x})\right]\right|}{\max _{\boldsymbol{\Omega}}\left|G_{m}(\boldsymbol{\Omega})\right|} .
$$

Thus (44) tends to select channels that are dominated by large amplitude components with frequency vectors lying near the maximum transmission frequency of the channel. This approach affords maximal rejection of cross-component interference and noise.

The dominant frequencies, which carry a rich description of the local texture structure, were termed emergent in [11]. Emergent image frequencies have been estimated previously by a constrained iterative relaxation procedure in [11] and by the TKEO in [8]. The advantages in using DCA are twofold. First, the frequency algorithms (25), (36), and (37) are local and computationally inexpensive. Second, DCA delivers signed frequency estimates, whereas the TKEO estimates unsigned frequency. In multiple dimensions, the relative signs of the components of the instantaneous frequency vector embody orientation, and are thus significant. DCA is primarily of interest in machine vision applications, where the dominant modulations are useful for solving a variety of problems including texture segmentation [11], [32], three-dimensional (3-D) surface reconstruction [11], [33], [34], and computational stereopsis [35]-[37].

\section{B. $C C A$}

CCA is depicted in the block diagram of Fig. 2. As in DCA, the filterbank channel responses are demodulated using (25) and (27) or (41)-(43). The component-wise decomposition of the signal is obtained by considering that the filterbank channels isolate components on a global basis. Modulating function estimates for one component in (1) are extracted from each filterbank channel. In 2-D applications, the filterbank used for CCA typically includes a baseband channel not present in Fig. 1. This is because the Fourier spectra of most images are dominated by the DC component, which is of little interest in DCA. In CCA, however, which seeks to compute image representations, incorporation of a baseband channel to capture visually important low frequency structure such as large scale shading and intensity variations is essential.

For an $M$-channel filterbank, CCA necessarily leads to an $M$-component computed model. The approach is inherently inefficient in this respect. In many cases, the AM functions of 


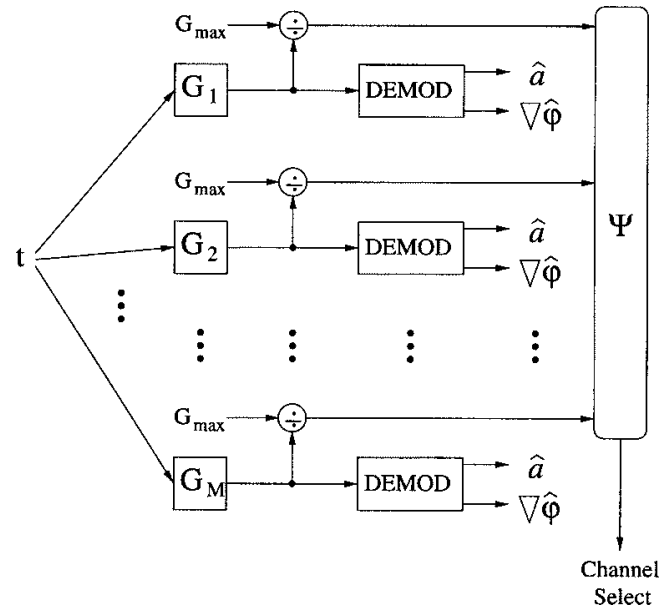

Fig. 1. Block diagram of DCA

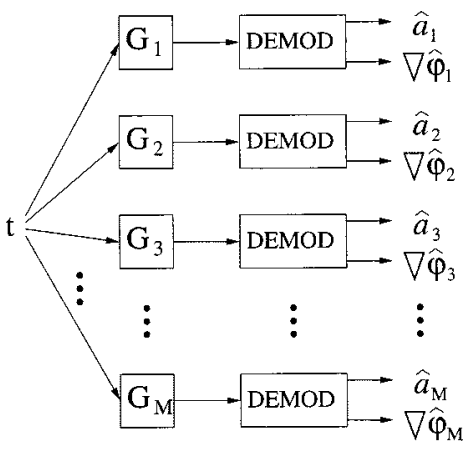

Fig. 2. Block diagram of CCA

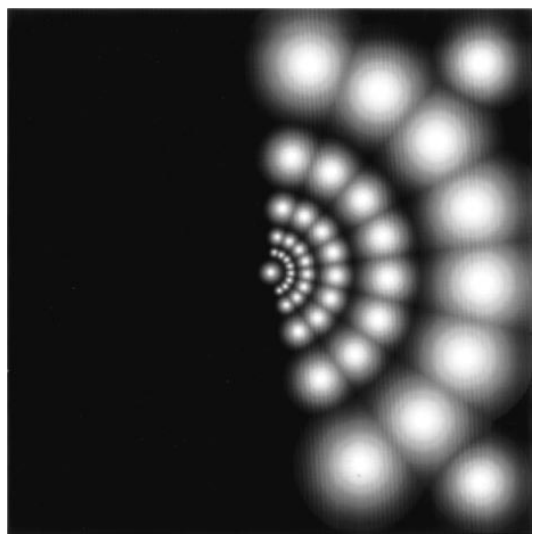

Fig. 3. Frequency domain depiction of multiband Gabor filterbank with baseband filter and two high frequency corner filters.

several channelized components are negligibly small over large regions. If nonorthogonal filters are used, as they often are to achieve good spatial localization without sacrificing a dense frequency covering, then certain elements of the signal structure are inevitably manifest redundantly in multiple channelized components. Furthermore, in regions where the signal structure is locally narrowband, the pass bands of many channels will lie far away from the signal frequencies. These channels are susceptible to noise and can produce modulating function estimates that are physically meaningless. In CCA signal reconstructions, the effects of these problems are generally most apparent over narrowband regions that are dominated by low fre- quencies. Such regions are characterized by relatively large spatial extent and a paucity of high frequency structure that might otherwise conceal the errors.

\section{Filterbank}

DCA and CCA are essentially independent of the particular filterbank that is used, and thus far we have made no specific assumptions about the filters except that they are of the LSI type. Clearly, they must be spectrally localized if they are to resolve multiple signal components from one another. They must also be spatially localized if they are to capture local nonstationary signal structure. Furthermore, in the continuous case, spatially localized filters generally admit small $p$-energy variances that lead to small approximation errors in (25) and (27). Spatially localized discrete filters tend to have small $\ell^{1}$-norms and also to yield small values for the energy functional (32). The desirability of spatially local discrete filters is made explicit in (30). These observations strongly suggest the use of multidimensional Gabor filters, which, in the continuous case, uniquely realize the uncertainty principle lower bound on simultaneous spatio-spectral localization. This optimal localization comes at a price, however. Since Gabor filterbanks are not orthogonal, their use in CCA generally leads to representation errors of the type discussed in Section IV-B when an image contains structure lying within frequency bands covered by the effective spectral support of multiple channel filters.

Typically, we use a bank of isotropic unity $L^{2}$-norm Gabor filters with half-peak radial bandwidths of one octave. The efficacy of such a filterbank for AM-FM demodulation has been well established [3], [11], [32], [38]. A 2-D Gabor filterbank of the type described in [11] is depicted in the frequency domain in Fig. 3, augmented by two high-frequency Gabor filters to cover the outermost corners of the right frequency half-plane. When the Hilbert transform (7) is taken in the horizontal direction, the Fourier spectra of complex signals generated by the technique described in Section II are supported only in the right frequency half-plane. The filterbank of Fig. 3 also incorporates a baseband channel for CCA and has 43 channels in total.

\section{Post Processing}

In estimating amplitude and frequency modulations from the responses of bandpass filters, post processing is generally required to compensate for approximation errors in the demodulation algorithm arising from signal perturbations that are not locally narrowband. Such signal perturbations were studied in detail in [38], where post-smoothing of the channel responses by low-pass filters having envelopes of the same shape as the channel filters themselves was shown to be effective. In the particular case of Gabor filters, it was recommended that each post filter space constant be designed 1.5 times larger than the space constant of the corresponding channel filter. In [1], low-pass postfiltering subsequent to bandpass Gabor filtering was used to compensate for approximation errors in the TKEO. Large spikes in the TKEO amplitude estimates were also smoothed by median filtering in [2].

It was demonstrated in [39] that input phase discontinuities can lead to unbounded excursions in the channel response instantaneous frequencies. Furthermore, natural images and 


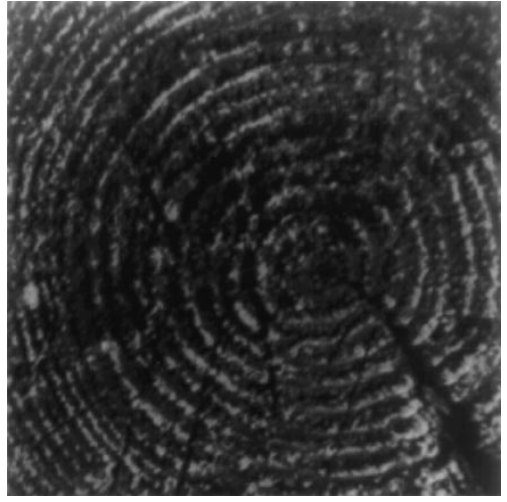

(a)

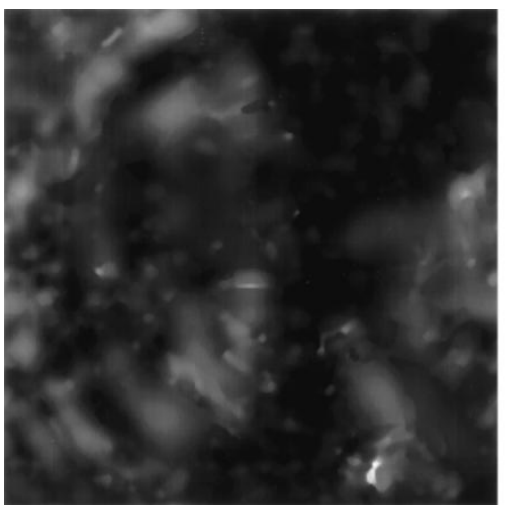

(c)

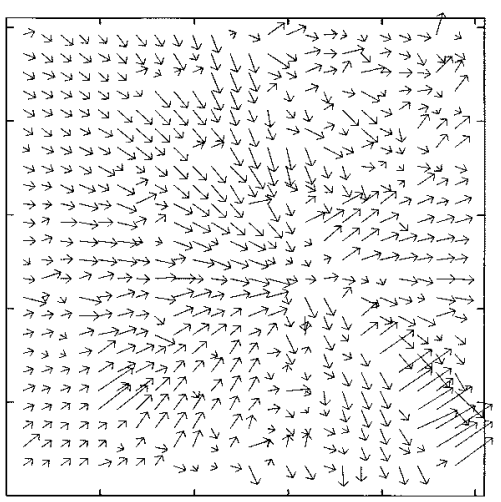

(b)

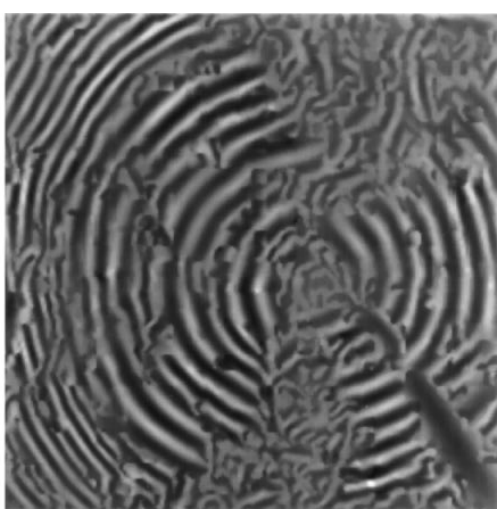

(d)

Fig. 4. DCA analysis of Tree image: (a) image, (b) needle diagram depicting estimated emergent frequencies (arrow length is proportional to the instantaneous period), (c) estimated dominant component amplitude modulation function, and (d) reconstruction of dominant component from estimated dominant modulations.

video generally contain phase discontinuities arising from a host of factors including occlusions, surface discontinuities, deformations and defects in surface topology, surface reflectance, shadows, specularities, and noise [32]. The effects of nonsmooth phase perturbations can be severe for DCA and CCA. The QEA's break down in the neighborhood of a phase discontinuity, and the frequency demodulation algorithms may suffer from large approximation errors. Because the frequency estimates appear in the denominators of (27) and (43), wideband frequency excursions and large frequency estimation errors generally lead to noise susceptibility and numerical instability that produce absurdly large amplitude estimates. The resulting increase in dynamic range can cause severe quantization errors when the signal is reconstructed.

For example, suppose that CCA is used to compute a multicomponent AM-FM representation for a gray scale image with eight-bit pixels taking values in the range $[0,255]$. Suppose further that the image contains phase discontinuities or other nonsmooth perturbations that lead to absurdly large amplitude estimates in the computed representation. Since the erroneous estimates often tend to be spatially localized, it is possible, and indeed even likely, that the computed representation will still be useful for machine vision applications. However, if one attempts to reconstruct the image from the representation, the erroneous amplitude estimates will generally cause the range of pixel values in the reconstructed image to be much larger than
$[0,255]$. If the reconstructed values are linearly mapped into the range $[0,255]$ for display, most of the accurately reconstructed image structure will be compressed into a dynamic range of only a few bits and lost.

As will be demonstrated in Section $\mathrm{V}$, we have found that post-smoothing of the channel responses is not effective for controlling this problem. Instead, our approach is to apply a low-pass Gaussian filter directly to the frequency estimates. The space constant of this Gaussian postfilter is set to a multiple of that of the channel filter, yielding a simple relationship between the postfilter and channel filter envelopes. We use the smoothed frequency estimates to compute the amplitudes, and then postfilter the amplitude estimates themselves. It is likely that other smoothing approaches such as median filtering would also be effective.

\section{EXAMPLES}

DCA and CCA were applied to several $256 \times 256$ gray scale images with eight bit pixels. In all cases, the (discretized) Hilbert transform (7) acted in the horizontal direction. A discrete version of the filterbank in Fig. 3 was used with Gaussian postfilters. Except where noted, the postfilter linear bandwidths were equal to those of the corresponding channel filters. For DCA, the baseband channel was eliminated from the filterbank. Reconstruction was performed using the algorithm given in 


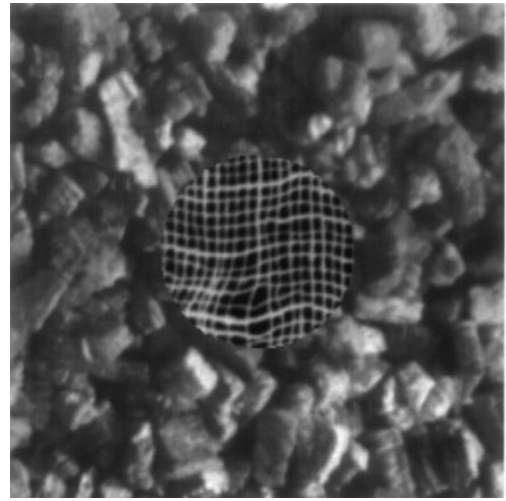

(a)

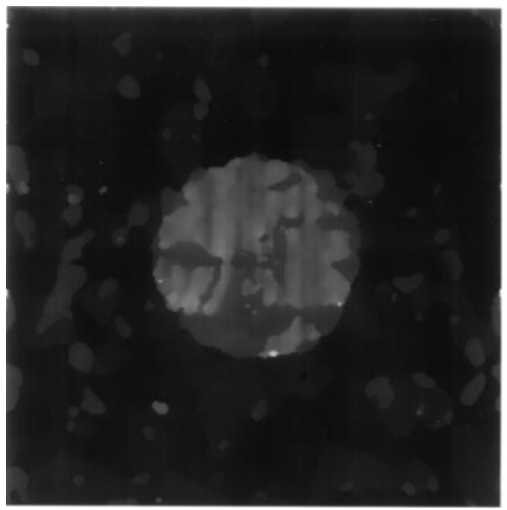

(c)

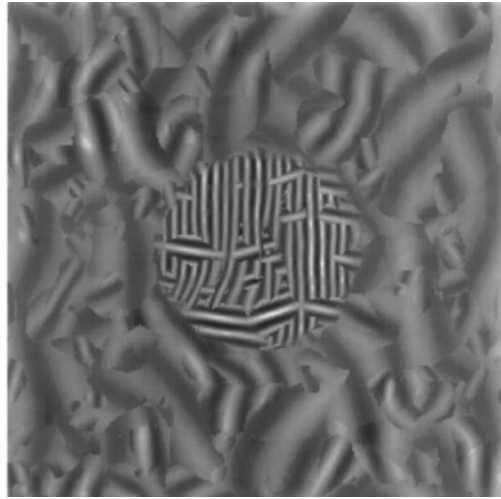

(b)

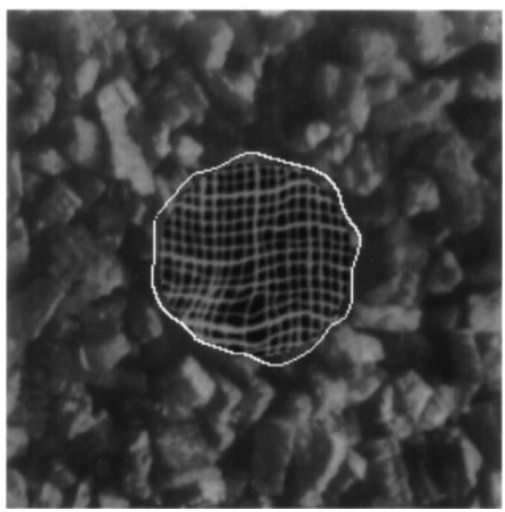

(d)

Fig. 5. DCA texture segmentation obtained by applying a $\nabla^{2} g$ edge detector to the estimated emergent frequency magnitudes: (a) Mica-Burlap image, (b) dominant component reconstruction, (c) estimated emergent frequency magnitudes displayed as a gray scale image, and (d) segmentation result.

[6] with a phase reconstruction interval of four pixels in each dimension.

DCA was first applied to the Tree image shown in Fig. 4(a). A needle diagram depicting the estimated emergent frequencies is given in Fig. 4(b), where needle length is inversely proportional to $\left|\nabla \hat{\varphi}_{D}(\boldsymbol{x})\right|$ (proportional to the instantaneous period). The estimated dominant AM function is shown in Fig. 4(c), and may be interpreted as contrast of the dominant component: bright areas in Fig. 4(c) generally correspond to regions of high contrast in Fig. 4(a). The dominant component reconstruction is given in Fig 4(d). The similarity between Fig. 4(d) and the original is striking, and suggests that much of the total image structure has been captured in a single AM-FM component.

Fig. 5 demonstrates the use of DCA to perform texture segmentation on the image Mica-Burlap shown in Fig. 5(a). The reconstructed dominant component appears in Fig. 5(b), and the estimated emergent frequency magnitudes are displayed as a gray scale image in Fig. 5(c). The patch-like regions that appear in Fig. 5(b) and (c) occur because different components are dominant in the different regions. The segmentation shown in Fig. 5(d) was obtained by applying a LoG edge detector with gradient magnitude thresholding [40] to the estimated emergent frequency magnitudes in Fig. 5(c).

The familiar image Mandrill appears in Fig. 6(a). Fig. 6(b) shows the dominant component reconstruction computed by DCA. CCA was also applied to obtain the 43-component recon- struction of Fig. 6(c). A reconstruction of one of the individual channelized components is given in Fig. 6(d). Fig. 6(e) shows the 43-component CCA reconstruction that is obtained in the absence of postfiltering. Because of the unmitigated effects of nonsmooth signal perturbations, almost no useful structure can be recovered from the computed modulation model in this case.

Fig. 6(f) and (g) also show 43-component CCA reconstructions, but with postfiltering applied to the channel responses instead of directly to the modulating function estimates. In Fig. 6(f), each postfilter space constant was two-thirds as large as that of the corresponding channel filter (our convention is to write the space constant $\sigma$ on the bottom in the space domain, so a smaller space constant results in a narrower impulse response and a wider spectrum). Although some useful structure has been recovered in Fig. 6(f), the overall quality is quite poor. In single precision floating point, the extremes of the original image were -1.0 and 0.7175 . Because of absurd amplitude estimates, the extremes of the floating point reconstruction in Fig. 6(f) were amplified to -167.9172 and 202.3926 . For the reconstruction of Fig. 6(g), the postfilter space constants were scaled by a factor of 1.5 as compared to those of the channel filters. In this case, the essential structure of the image was successfully recovered from the computed modulation model. Significant detail was lost due to the excessive postfiltering, however, and numerous amplitude spikes are still clearly visible. In single precision floating point, the extremes of the 


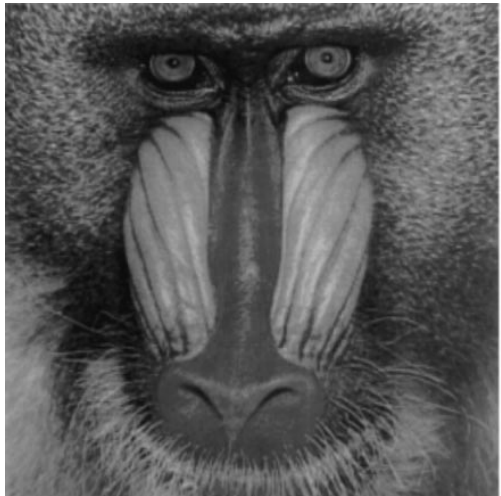

(a)

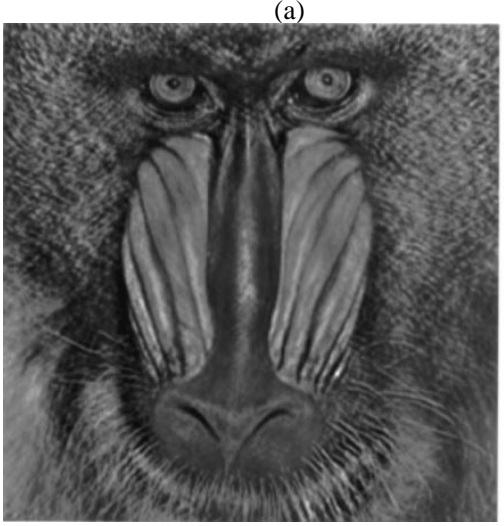

(c)

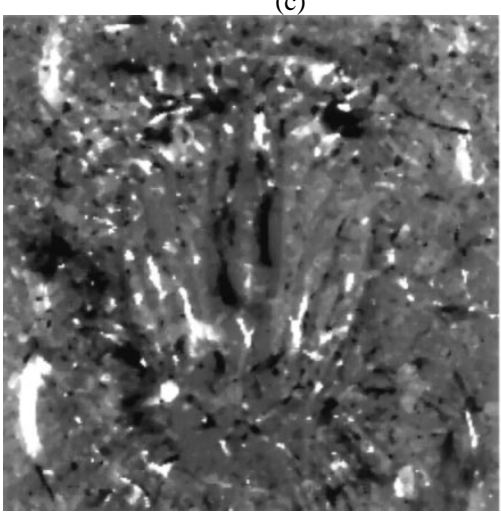

(e)

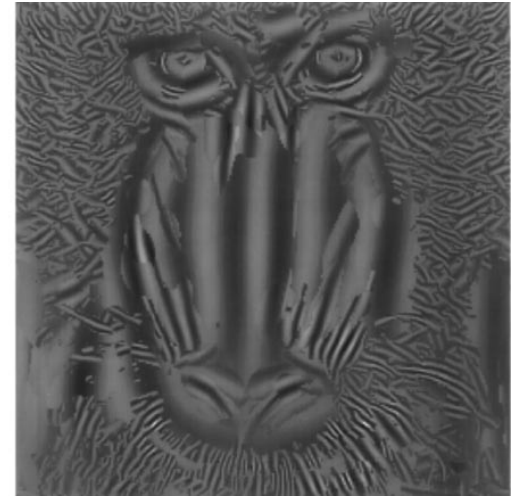

(b)

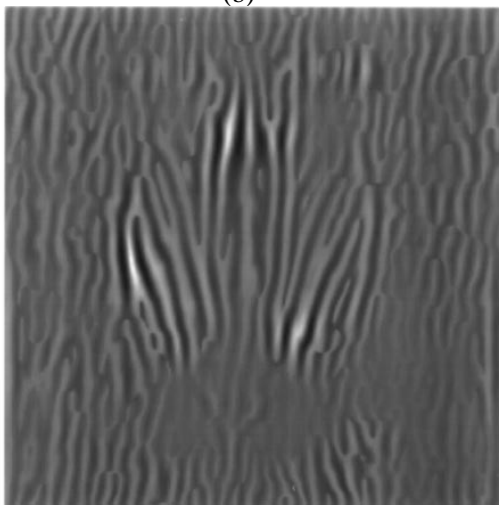

(d)

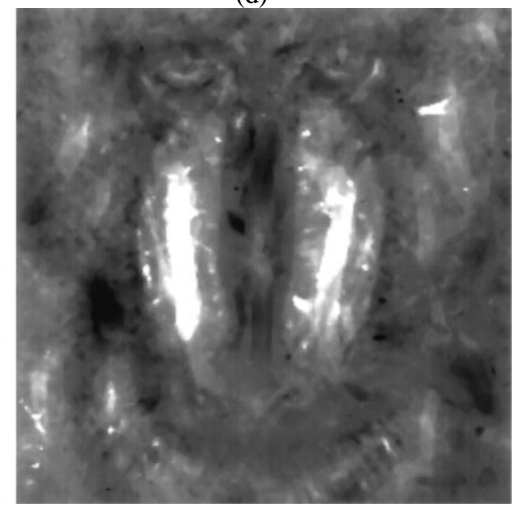

(f)

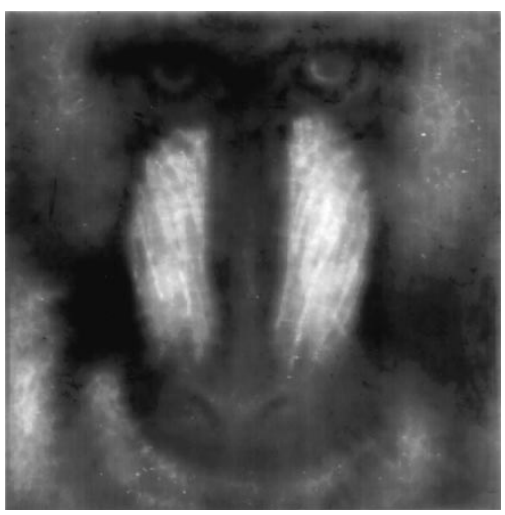

(g)

Fig. 6. AM-FM representation of Mandrill image: (a) image, (b) dominant component reconstruction, (c) reconstruction from 43 channelized components, (d) reconstruction of one channelized component, (e) 43-component CCA reconstruction without postfiltering, (f) 43-component CCA reconstruction with postfiltering applied to channel responses; $\sigma$ is scaled by $2 / 3$, and (g) 43-component CCA reconstruction with postfiltering applied to channel responses; $\sigma$ is scaled by 1.5 . 


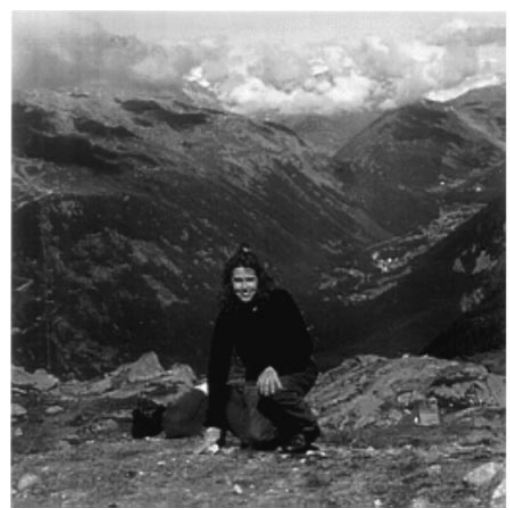

(a)

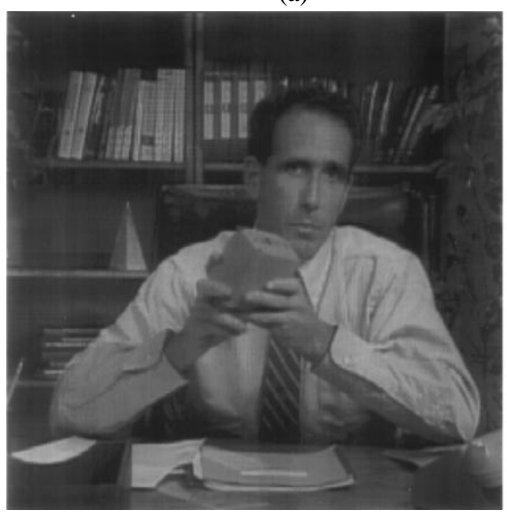

(c)

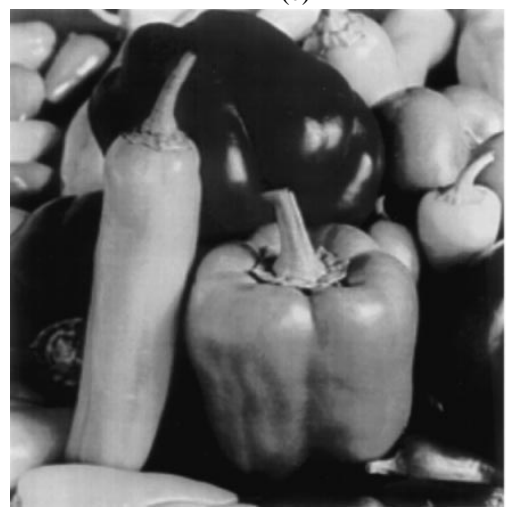

(e)

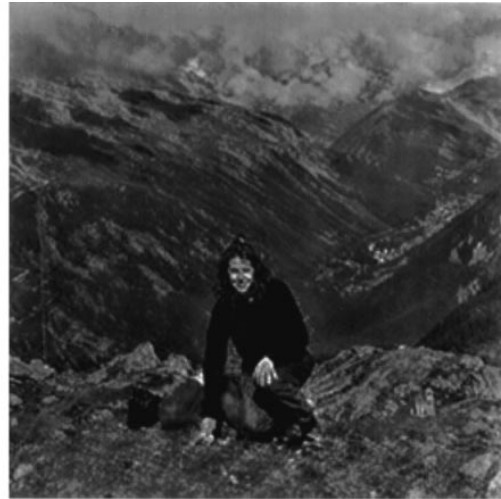

(b)

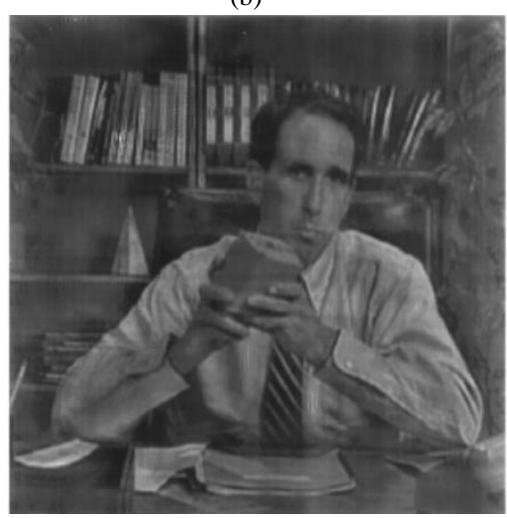

(d)

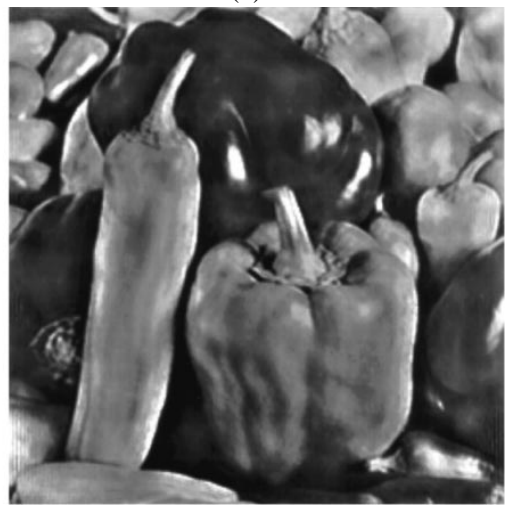

(f)

Fig. 7. Channelized component analysis: (a) Zermatt image, (b) CCA reconstruction, (c) Salesman image, (d) CCA reconstruction, (e) Peppers image, and (f) CCA reconstruction.

reconstruction in Fig. 6(g) were -39.0345 and 19.0505. The results of setting the postfilter and channel filter space constants equal were not unlike those shown in Fig. 6(f).

In Fig. 7, CCA was applied to the images Zermatt, Salesman, and Peppers. Although the reconstructions are in good perceptual agreement with the originals, the MAE is on the order of 100 (out of 255) in each case. The most noticeable errors appear to be contrast distortions occurring in regions that are dominated by low-frequency structure. Finally, it is interesting to take note of the small bright dot appearing along the top left edge of the Salesman image. The CCA reconstruction of this dot exhibits ringing artifacts reminiscent of those commonly seen in linear image restoration and in many wavelet-based signal representation algorithms. Similar ringing artifacts are visible along the left and right edges of the Peppers reconstruction in Fig. 7(f).

\section{CONCLUSION}

This paper addressed the problem of modeling sophisticated multidimensional signals as multicomponent sums of locally narrowband AM-FM functions. Working in a general $n$-dimensional framework, we developed powerful approximations that express the responses of LSI systems to AM-FM inputs directly in terms of the input modulating functions. This is significant because a closed form solution for the response cannot be obtained due to the inherently nonlinear interaction between the modulating functions and the system impulse response. We bounded the errors in these quasi-eigenfunction approximations by generalized energy variances quantifying the spatial concentration of the filter and by the local smoothness of the modulating functions expressed as Sobolev norms in the continuous case and path and line integrals in the discrete case. 
We applied the approximations to derive novel nonlinear multidimensional demodulation algorithms capable of obtaining estimates of a signal's amplitude and frequency modulations from the channel responses of a multiband linear filterbank. Two practical techniques for computing multidimensional AM-FM models for multicomponent signals were introduced and demonstrated. Dominant component analysis (DCA) delivers a single pair of modulating functions that characterize the dominant signal structure on a spatially local basis. In addition to being useful for general nonstationary analysis, the dominant modulations form the basis for a variety of texture-based approaches to the solution of classical problems in machine vision. Channelized components analysis (CCA) is a true multidimensional multicomponent technique capable of delivering rich signal representations in the modulation domain. It is particularly novel in view of the paucity of competing methods.

The CCA examples presented in Section $\mathrm{V}$ are the first to demonstrate multidimensional multicomponent AM-FM modeling and reconstruction for the types of images that are of practical interest in broad application areas. Consequently, a number of important questions remain to be answered by future research. The computation of multicomponent AM-FM models is inherently ill-posed, both with respect to the decomposition of a real-valued multipartite signal into components and with respect to the demodulation of those components. The Gabor filterbank used in this work was chosen for its attractive localization properties. It is entirely possible that improved results could be obtained using orthogonal filterbanks or alternative filter types designed to minimize the ringing artifacts and contrast distortions visible in Figs. 6 and 7. Nonlinear regression techniques may also prove useful for decomposing a signal into components; unfiltered demodulation algorithms such as (36) and (37) could then be applied directly on a component-wise basis. In addition, improved postfiltering and reconstruction techniques merit considerable future investigation. Finally, the development of new and meaningful criteria for evaluating the performance of multicomponent AM-FM models is another important open problem.

\section{APPENDIX}

\section{Proof of Corollary 1: Define}

$$
\begin{aligned}
& A(\boldsymbol{x})=a_{x_{i}}^{+}(\boldsymbol{x}) \exp [j \varphi(\boldsymbol{x})], \quad B(\boldsymbol{x})=a_{x_{i}}^{-}(\boldsymbol{x}) \exp [j \varphi(\boldsymbol{x})] \\
& C(\boldsymbol{x})=a(\boldsymbol{x}) \varphi_{x_{i}}^{+}(\boldsymbol{x}) \exp [j \varphi(\boldsymbol{x})]
\end{aligned}
$$

and

$$
D(\boldsymbol{x})=a(\boldsymbol{x}) \varphi_{x_{i}}^{-}(\boldsymbol{x}) \exp [j \varphi(\boldsymbol{x})] .
$$

Then

$$
t_{x_{i}}(\boldsymbol{x})=A(\boldsymbol{x})-B(\boldsymbol{x})+j C(\boldsymbol{x})-j D(\boldsymbol{x}) .
$$

For $A(\boldsymbol{x})$, define

$$
\psi_{i, A}(\boldsymbol{x})=g(\boldsymbol{x}) * A(\boldsymbol{x}), \quad \hat{\psi}_{i, A}(\boldsymbol{x})=A(\boldsymbol{x}) G[\nabla \varphi(\boldsymbol{x})]
$$

and

$$
\varepsilon_{A}(\boldsymbol{x})=\left|\psi_{i, A}(\boldsymbol{x})-\hat{\psi}_{i, A}(\boldsymbol{x})\right|
$$

Make similar definitions for $B(\boldsymbol{x}), C(\boldsymbol{x})$, and $D(\boldsymbol{x})$. Since $g(\boldsymbol{x})$ is linear, it follows from (A.1) that

$$
\psi_{i}(\boldsymbol{x})=\psi_{i, A}(\boldsymbol{x})-\psi_{i, B}(\boldsymbol{x})+j \psi_{i, C}(\boldsymbol{x})-j \psi_{i, D}(\boldsymbol{x}) .
$$

Direct substitution of (A.1) into the right side of (16) yields

$$
\hat{\psi}_{i}(\boldsymbol{x})=\hat{\psi}_{i, A}(\boldsymbol{x})-\hat{\psi}_{i, B}(\boldsymbol{x})+j \hat{\psi}_{i, C}(\boldsymbol{x})-j \hat{\psi}_{i, D}(\boldsymbol{x}) \text {. }
$$

By subtracting (A.3) from (A.2), taking the magnitude of the difference, and applying the triangle inequality, we bound $\varepsilon_{\psi_{i}}(\boldsymbol{x})$ according to

$$
\varepsilon_{\psi_{i}}(\boldsymbol{x}) \leq \varepsilon_{A}(\boldsymbol{x})+\varepsilon_{B}(\boldsymbol{x})+\varepsilon_{C}(\boldsymbol{x})+\varepsilon_{D}(\boldsymbol{x}) .
$$

Applying Theorem 1 to $\varepsilon_{A}(\boldsymbol{x})$, we obtain

$$
\begin{aligned}
\varepsilon_{A}(\boldsymbol{x}) \leq & \frac{q}{q-n} \overline{\boldsymbol{\Delta}^{p}}(g) \overline{\mathcal{D}^{q}}\left(a_{x_{i}}^{+}\right) \\
& +\frac{q^{2} a_{\max }}{(q-n)(2 q-n)} \overline{\boldsymbol{\Theta}^{p}}(g) \overline{\mathcal{S}^{q}}(\varphi) .
\end{aligned}
$$

The result (18) follows immediately upon applying Theorem 1 to $\varepsilon_{B}(\boldsymbol{x}), \varepsilon_{C}(\boldsymbol{x})$, and $\varepsilon_{D}(\boldsymbol{x})$ in (A.4) and collecting terms.

Proof of Theorem 2: Expanding $\varphi(\boldsymbol{x})$ in a first-order Taylor series with explicit remainder [41], we obtain

$$
\varphi(\boldsymbol{k}-\boldsymbol{p})=\varphi(\boldsymbol{k})-\boldsymbol{p}^{T} \nabla \varphi(\boldsymbol{k})+Q_{\varphi}(\boldsymbol{k}, \boldsymbol{p})
$$

where

$$
Q_{\varphi}(k, \boldsymbol{p})=\int_{0}^{1}(1-s) \boldsymbol{p}^{T}\left\langle\boldsymbol{p}^{T} \nabla \varphi_{x_{i}}(\boldsymbol{k}-s \boldsymbol{p})\right\rangle d s .
$$

Substitution of $t(\boldsymbol{k}-\boldsymbol{p})=a(\boldsymbol{k}-\boldsymbol{p}) \exp [j \varphi(\boldsymbol{k}-\boldsymbol{p})]$ and (A.5) into (28) then yields

$$
\begin{aligned}
y(\boldsymbol{k})= & \sum_{\boldsymbol{p} \in \mathbb{Z}^{n}} g(\boldsymbol{p}) a(\boldsymbol{k}-\boldsymbol{p}) \exp [j \varphi(\boldsymbol{k})] \\
& \cdot \exp \left[-j \boldsymbol{p}^{T} \nabla \varphi(\boldsymbol{k})\right] \exp \left[j Q_{\varphi}(\boldsymbol{k}, \boldsymbol{p})\right] .
\end{aligned}
$$

Upon replacing $G(\cdot)$ in (29) with the definition of the Fourier transform of a sequence [42], we have

$$
\begin{aligned}
\hat{y}(\boldsymbol{k}) & =\left.t(\boldsymbol{k}) \sum_{\boldsymbol{p} \in \mathbb{Z}^{n}} g(\boldsymbol{p}) \exp \left[-j \boldsymbol{p}^{T} \boldsymbol{\omega}\right]\right|_{\boldsymbol{\omega}=\nabla \varphi(\boldsymbol{k})} \\
& =\sum_{\boldsymbol{p} \in \mathbb{Z}^{n}} g(\boldsymbol{p}) a(\boldsymbol{k}) \exp [j \varphi(\boldsymbol{k})] \exp \left[-j \boldsymbol{p}^{T} \nabla \varphi(\boldsymbol{k})\right] .
\end{aligned}
$$

Now, we write the QEA error $\varepsilon_{y}(\boldsymbol{k})=|y(\boldsymbol{k})-\hat{y}(\boldsymbol{k})|$ in terms of (A.7) and (A.8), apply the triangle inequality, and finally apply the inequality $|\alpha-\beta| \leq|\alpha-z|+|z-\beta|$ [43] to obtain

$$
\begin{aligned}
\varepsilon_{y}(\boldsymbol{k}) \leq & \sum_{\boldsymbol{p} \in \mathbb{Z}^{n}}|g(\boldsymbol{p})|\left|a(\boldsymbol{k}-\boldsymbol{p}) \exp \left[j Q_{\varphi}(\boldsymbol{k}, \boldsymbol{p})\right]-a(\boldsymbol{k})\right| \\
\leq & \sum_{\boldsymbol{p} \in \mathbb{Z}^{n}}|g(\boldsymbol{p})|\{|a(\boldsymbol{k}-\boldsymbol{p})-a(\boldsymbol{k})| \\
& \left.+a(\boldsymbol{k}-\boldsymbol{p})\left|\exp \left[j Q_{\varphi}(\boldsymbol{k}, \boldsymbol{p})\right]-1\right|\right\} \\
\leq & \sum_{\boldsymbol{p} \in \mathbb{Z}^{n}}|g(\boldsymbol{p})|\{|a(\boldsymbol{k}-\boldsymbol{p})-a(\boldsymbol{k})| \\
& \left.+a_{\max }\left|\exp \left[j Q_{\varphi}(\boldsymbol{k}, \boldsymbol{p})\right]-1\right|\right\}
\end{aligned}
$$


whereupon application of the inequality $\left|e^{j \theta}-1\right| \leq|\theta|, \theta \in \mathbb{R}$, gives

$\varepsilon_{y}(\boldsymbol{k}) \leq \sum_{\boldsymbol{p} \in \mathbb{Z}^{n}}|g(\boldsymbol{p})|\left\{|a(\boldsymbol{k}-\boldsymbol{p})-a(\boldsymbol{k})|+a_{\max }\left|Q_{\varphi}(\boldsymbol{k}, \boldsymbol{p})\right|\right\}$.

Taking the magnitude of (A.6), applying the triangle inequality, and observing that $|1-s| \leq 1 \forall s \in[0,1]$, we verify that

$$
\begin{aligned}
\left|Q_{\varphi}(\boldsymbol{k}, \boldsymbol{p})\right| & \leq \int_{0}^{1}|1-s|\left|\boldsymbol{p}^{T}\left\langle\boldsymbol{p}^{T} \nabla \varphi_{x_{i}}(\boldsymbol{k}-s \boldsymbol{p})\right\rangle\right| d s \\
& \leq \int_{0}^{1}\left|\boldsymbol{p}^{T}\left\langle\boldsymbol{p}^{T} \nabla \varphi_{x_{i}}(k-s \boldsymbol{p})\right\rangle\right| d s
\end{aligned}
$$

The proof is completed by further observing that $\left|Q_{\varphi}(\boldsymbol{k}, \boldsymbol{p})\right|=$ 0 and $|a(\boldsymbol{k}-\boldsymbol{p})-a(\boldsymbol{k})|=\mathbf{0}$ when $\boldsymbol{p}=\mathbf{0}$, and then substituting (A.10) back into (A.9).

Proof of Corollary 2: We begin by isolating the contributions of amplitude and phase in the approximation error. Distributing multiplication over addition under the sum in (A.9), we have

$$
\begin{aligned}
\varepsilon_{y}(\boldsymbol{k}) \leq & \underbrace{\sum_{\boldsymbol{p} \in \mathbb{Z}^{\imath 2}}|g(\boldsymbol{p})||a(\boldsymbol{k}-\boldsymbol{p})-a(\boldsymbol{k})|}_{\varepsilon_{y, a}(\boldsymbol{k})} \\
& +a_{\max } \underbrace{\sum_{\boldsymbol{p} \in \mathbb{Z}^{n}}|g(\boldsymbol{p})|\left|Q_{\varphi}(\boldsymbol{k}, \boldsymbol{p})\right|}_{\varepsilon_{y, \varphi}(\boldsymbol{k})} \\
& \triangleq \varepsilon_{\boldsymbol{y}, a}(\boldsymbol{k})+a_{\max } \varepsilon_{\boldsymbol{y}, \varphi}(\boldsymbol{k})
\end{aligned}
$$

where (A.12) defines $\varepsilon_{y, a}(\boldsymbol{k})$ and $\varepsilon_{y, \varphi}(\boldsymbol{k})$. For $s \in[0,1]$, let $\boldsymbol{\mu}_{\boldsymbol{k}, \boldsymbol{p}}(s)=\boldsymbol{k}-s \boldsymbol{p}$. Application of the first form of the fundamental theorem of calculus then gives

$$
\int_{\boldsymbol{\mu}_{\boldsymbol{k}, \boldsymbol{p}}} \nabla a(\boldsymbol{x}) \cdot d \boldsymbol{x}=a(\boldsymbol{k})-a(\boldsymbol{k}-\boldsymbol{p})
$$

so

$$
|a(k-\boldsymbol{p})-a(\boldsymbol{k})|=\left|\int_{\boldsymbol{\mu}_{\boldsymbol{k}, \boldsymbol{p}}} \nabla a(\boldsymbol{x}) \cdot d \boldsymbol{x}\right|
$$

Substituting this result into the expression for $\varepsilon_{y, a}(k)$ in (A.11) and observing that (A.13) is identically zero when $\boldsymbol{p}=\mathbf{0}$, we obtain

$$
\begin{aligned}
\varepsilon_{y, a}(\boldsymbol{k}) & =\sum_{\substack{\boldsymbol{p} \in \mathbb{Z}^{n} \\
\boldsymbol{p} \neq \mathbf{0}}}|g(\boldsymbol{p})|\left|\int_{\boldsymbol{\mu}_{\boldsymbol{k}, \boldsymbol{p}}} \nabla a(\boldsymbol{x}) \cdot d \boldsymbol{x}\right| \\
& \leq \sup _{\boldsymbol{\sigma} \in P^{1}}\left|\int_{\boldsymbol{\sigma}} \nabla a(\boldsymbol{x}) \cdot d \boldsymbol{x}\right| \sum_{\substack{\boldsymbol{p} \in \mathbb{Z}^{n} \\
\boldsymbol{p} \neq \mathbf{0}}}|g(\boldsymbol{p})| \\
& =\left\{\|g\|_{\ell^{1}}-|g(\mathbf{0})|\right\} \mathfrak{L}^{1}(a) .
\end{aligned}
$$

Upon substitution of (A.6) into the expression (A.11) for $\varepsilon_{y, \varphi}(k)$ and alternating between angle bracket notation and sums, we have that

$$
\begin{aligned}
& \varepsilon_{y, \varphi}(\boldsymbol{k}) \leq \sum_{\substack{\boldsymbol{p} \in \mathbb{Z}^{n} \\
\boldsymbol{p} \neq 0}}|g(\boldsymbol{p})| \\
& \cdot\left|\int_{0}^{1}(1-s) \sum_{i=1}^{n} \sum_{m=1}^{n} p_{i} p_{m} \varphi_{x_{i}, x_{m}}(\boldsymbol{k}-s \boldsymbol{p}) d s\right| \\
& \leq \sum_{\substack{\boldsymbol{p} \in \mathbb{Z}^{n} \\
\boldsymbol{p} \neq \mathbf{0}}} \sum_{i=1}^{n}|g(\boldsymbol{p})| \\
& \cdot\left|\int_{0}^{1}(1-s) p_{i} \sum_{m=1}^{n} p_{m} \varphi_{x_{i}, x_{m}}(k-s \boldsymbol{p}) d s\right| \\
& =\sum_{\substack{\boldsymbol{p} \in \mathbb{Z}^{n} \\
\boldsymbol{p} \neq 0}} \sum_{i=1}^{n}|g(\boldsymbol{p})| \\
& \cdot\left|\int_{0}^{1}(1-s) p_{i} \boldsymbol{p}^{T} \nabla \varphi_{x_{i}}(\boldsymbol{k}-s \boldsymbol{p}) d s\right| .
\end{aligned}
$$

Now, for each $i \in[1, n]$, the integrand in (A.15) is zero $\forall \boldsymbol{p}$ such that $p_{i}=0$. For the remaining terms of the sum we have that $p_{i} \neq 0$ and $\boldsymbol{p} \neq \mathbf{0}$. Then $\left|p_{i}\right||\boldsymbol{p}| \neq 0$, and hence

$$
\begin{aligned}
\varepsilon_{y, \varphi}(\boldsymbol{k}) \leq & \sum_{\substack{\boldsymbol{p} \in \mathbb{Z}^{\eta} \\
\boldsymbol{p} \neq 0}} \sum_{\substack{i \in[1, n] \\
p_{i} \neq 0}}\left|p_{i}\right||\boldsymbol{p}||g(\boldsymbol{p})| \\
& \cdot\left|\int_{0}^{1}(1-s) \frac{p_{i} \boldsymbol{p}^{T}}{\left|p_{i}\right||\boldsymbol{p}|} \nabla \varphi_{x_{i}}(\boldsymbol{k}-s \boldsymbol{p}) d s\right| .
\end{aligned}
$$

For each $\boldsymbol{p} \in \mathbb{Z}^{n}$ such that $\boldsymbol{p} \neq \mathbf{0}$ and for each $i \in[1, n]$ such that $p_{i} \neq 0$, let

$$
\boldsymbol{u}_{i}(\boldsymbol{p})=\frac{p_{i} \boldsymbol{p}}{\left|p_{i}\right||\boldsymbol{p}|} .
$$

Clearly, $u_{i}(\boldsymbol{p})$ is a unit vector. Upon applying the triangle inequality, observing that $|1-s| \leq 1 \forall s \in[0,1]$, and interchanging the order of summation in (A.16), there results

$$
\begin{aligned}
& \varepsilon_{y, \varphi}(\boldsymbol{k}) \leq \sum_{\substack{\boldsymbol{p} \in \mathbb{Z}^{n} \\
\boldsymbol{p} \neq \mathbf{0}}} \sum_{\substack{i \in[1, n] \\
p_{i} \neq 0}}\left|p_{i}\right||\boldsymbol{p}||g(\boldsymbol{p})| \\
& \cdot \int_{0}^{1}|1-s|\left|\boldsymbol{u}_{i}^{T}(\boldsymbol{p}) \nabla \varphi_{x_{i}}(\boldsymbol{k}-\boldsymbol{s} \boldsymbol{p})\right| d s \\
& \leq \sum_{\substack{\boldsymbol{p} \in \mathbb{Z}^{n} \\
\boldsymbol{p} \neq \mathbf{0}}} \sum_{\substack{i \in[1, n] \\
p_{i} \neq 0}}\left|p_{i}\right||\boldsymbol{p}||g(\boldsymbol{p})| \\
& \cdot \int_{\boldsymbol{\mu}_{\boldsymbol{k}, \boldsymbol{p}}}\left|\nabla \varphi_{x_{i}}(\boldsymbol{x})\right| d s \\
& \leq \sum_{i=1}^{n} \mathfrak{S}_{i}^{1}(\varphi) \sum_{\substack{\boldsymbol{p} \in \mathbb{Z}^{n} \\
\boldsymbol{p} \neq 0}}\left|\boldsymbol{p}^{T} \boldsymbol{e}_{i}^{T} \boldsymbol{p}\right||g(\boldsymbol{p})| \\
& =\sum_{i=1}^{n} \mathfrak{A}_{i}(g) \mathfrak{S}_{i}^{1}(\varphi) \\
& =\left\langle\mathfrak{A}_{i}(g)\right\rangle^{T}\left\langle\mathfrak{S}_{i}^{1}(\varphi)\right\rangle \text {. }
\end{aligned}
$$


Substitution of (A.14) and (A.17) into (A.12) immediately yields the desired result (31).

\section{ACKNOWLEDGMENT}

The authors would like to thank the three anonymous reviewers whose comments and suggestions greatly improved the paper.

\section{REFERENCES}

[1] P. Maragos, J. F. Kaiser, and T. F. Quatieri, "On amplitude and frequency demodulation using energy operators," IEEE Trans. Signal Processing, vol. 41, pp. 1532-1550, Apr. 1993.

[2] — , "Energy separation in signal modulations with applications to speech analysis," IEEE Trans. Signal Processing, vol. 41, pp. 3024-3051, Oct. 1993.

[3] A. C. Bovik, P. Maragos, and T. F. Quatieri, "AM-FM energy detection and separation in noise using multiband energy operators," IEEE Trans. Signal Processing, vol. 41, pp. 3245-3265, Dec. 1993.

[4] H. M. Hanson, P. Maragos, and A. Potamianos, "A system for finding speech formants and modulations via energy separation," IEEE Trans. Speech Audio Processing, vol. 2, no. 3, pp. 436-443, July 1994.

[5] S. Lu and P. C. Doerschuk, "Nonlinear modeling and processing of speech based on sums of AM-FM formant models," IEEE Trans. Signal Processing, vol. 44, pp. 773-782, Apr. 1996.

[6] J. P. Havlicek, D. S. Harding, and A. C. Bovik, "The multi-component AM-FM image representation," IEEE Trans. Image Processing, vol. 5, pp. 1094-1100, June 1996.

[7] B. Friedlander and J. M. Francos, "An estimation algorithm for 2-D polynomial phase signals," IEEE Trans. Image Processing, vol. 5, pp. 1084-1087, June 1996.

[8] P. Maragos and A. C. Bovik, "Image demodulation using multidimensional energy separation," J. Opt. Soc. Amer. A, vol. 12, pp. 1867-1876, Sept. 1995.

[9] S. K. Mitra, S. Thurnhofer, M. Lightstone, and N. Strobel, "Two-dimensional Teager operators and their image processing applications," in Proc. 1995 IEEE Workshop Nonlin. Signal Image Processing, Halkidiki, Greece, June 20-22, 1995, pp. 959-962.

[10] H. Knutsson, C. F. Westin, and G. Granlund, "Local multiscale frequency and bandwidth estimation," in Proc. IEEE Int. Conf. Image Processing, vol. I, Austin, TX, 1994, pp. 36-40.

[11] A. C. Bovik, N. Gopal, T. Emmoth, and A. Restrepo, "Localized measurement of emergent image frequencies by Gabor wavelets," IEEE Trans. Inform. Theory, vol. 38, pp. 691-712, Mar. 1992.

[12] T.-H. Yu, S. K. Mitra, and J. F. Kaiser, "Novel algorithm for image enhancement," in Proc. SPIE/SPSE Conf. Image Proc. Alg. Tech. II, San Jose, CA, Feb. 1991.

[13] D. Wei and A. C. Bovik, "On the instantaneous frequencies of multicomponent AM-FM signals," IEEE Signal Processing Lett., vol. 5, pp. 84-86, Apr. 1998.

[14] A. C. Bovik, J. P. Havlicek, D. S. Harding, and M. D. Desai, "Limits on discrete modulated signals," IEEE Trans. Signal Processing, vol. 45, pp. 867-879, Apr. 1997.

[15] L. Cohen, Time-Frequency Analysis. Englewood Cliffs, NJ: PrenticeHall, 1995.

[16] B. Santhanam and P. Maragos, "Energy demodulation of two-component AM-FM signal mixtures," IEEE Signal Processing Lett., vol. 3, pp. 294-298, Nov. 1996.

[17] B. Friedlander and J. M. Francos, "Estimation of amplitude and phase parameters of multicomponent signals," IEEE Trans. Signal Processing, vol. 43, pp. 917-926, Apr. 1995.

[18] J. P. Havlicek, D. S. Harding, and A. C. Bovik, "Extracting essential modulated image structure," in Proc. 30th IEEE Asilomar Conf. Signals, Syst., Comput., Pacific Grove, CA, Nov. 3-6, 1996, pp. 1014-1018.
[19] S. Lu and P. C. Doerschuk, "Demodulators for AM-FM models of speech signals: A comparison," in Proc. IEEE Int. Conf. Acoust., Speech, Signal Proc., Atlanta, GA, May 7-10, 1996, pp. 263-266.

[20] J. P. Havlicek, D. S. Harding, and A. C. Bovik, "Multi-component signal demodulation and reconstruction using AM-FM modulation models," in Proc. 1995 IEEE Workshop Nonlin. Signal Image Processing, Halkidiki, Greece, June 20-22, 1995.

[21] J. P. Havlicek, J. W. Havlicek, and A. C. Bovik, "The analytic image," in Proc. IEEE Int. Conf. Image Processing, Santa Barbara, CA, Oct. 26-29, 1997.

[22] F. Peyrin, Y. M. Zhu, and R. Goutte et al., "Extension of the notion of analytic signal for multidimensional signals. Application to images," in Signal Processing III: Theories and Applications, I. T. Young et al., Eds. Amsterdam, The Netherlands: Elsevier, 1986, pp. 677-680.

[23] E. M. Stein, Singular Integrals and Differentiability Properties of Functions. Princeton, NJ: Princeton Univ. Press, 1970.

[24] D. Gabor, "Theory of communication," J. Inst. Elect. Eng. Lond., vol. 93, no. III, pp. 429-457, 1946.

[25] J. Ville, "Théorie et applications de la notation de signal analytique," Cables Transm., vol. 2A, pp. 61-74, 1948. Translated to English in I. Selin, "Theory and applications of the notion of complex signal," Tech. Rep. T-92, RAND Corp., Santa Monica, CA, Aug. 1958..

[26] D. Vakman, "On the analytic signal, the Teager-Kaiser energy algorithm, and other methods for defining amplitude and frequency," IEEE Trans. Signal Processing, vol. 44, pp. 791-797, Apr. 1996.

[27] V. Čižek, "Discrete Hilbert transform," IEEE Trans. Audio Electroacoust., vol. AU-18, pp. 340-343, Dec. 1970.

[28] Y. M. Zhu, F. Peyrin, and R. Goutte, "The use of a two-dimensional Hilbert transform for Wigner analysis of 2-dimensional real signals," Signal Process., vol. 19, pp. 205-220, 1990.

[29] R. R. Read and S. Treitel, "The stabilization of two-dimensional recursive filters via the discrete Hilbert transform," IEEE Trans. Geosci. Electron., vol. GE-11, no. 3, pp. 153-160, 1973.

[30] S. L. Hahn, "Multidimensional complex signals with single-orthant spectra," Proc. IEEE, vol. 80, pp. 1287-1300, Aug. 1992.

[31] J. P. Havlicek and A. C. Bovik, "AM-FM models, the analytic image, and nonlinear demodulation techniques," Center for Vision and Image Sci., Univ. Texas at Austin, Tech. Rep. TR-95-001, Mar. 1995.

[32] A. C. Bovik, M. Clark, and W. S. Geisler, "Multichannel texture analysis using localized spatial filters," IEEE Trans. Pattern Anal. Machine Intell., vol. 12, pp. 55-73, Jan. 1990.

[33] B. J. Super and A. C. Bovik, "Planar surface orientation from texture spatial frequencies," Pattern Recognit., vol. 28, no. 5, pp. 728-743, 1995.

[34] — "Shape from texture using local spectral moments," IEEE Trans. Pattern Anal. Machine Intell., vol. 17, pp. 333-343, Apr. 1995.

[35] T.-Y. Chen and A. C. Bovik, "Stereo disparity from multiscale processing of local image phase," in Proc. IEEE Int. Symp. Comput. Vision, Coral Gables, FL, Nov. 20-22, 1995.

[36] T.-Y. Chen, A. C. Bovik, and B. J. Super, "Multiscale stereopsis via Gabor filter phase response," in Proc. IEEE Int. Conf. Syst., Man, Cybern., San Antonio, TX, Oct. 2-5, 1994, pp. 55-60.

[37] T.-Y. Chen, A. C. Bovik, and L. K. Cormack, "Stereoscopic ranging by matching image modulations," IEEE Trans. Image Processing, vol. 8, pp. 785-797, June 1999.

[38] A. C. Bovik, "Analysis of multichannel narrow-band filters for image texture segmentation," IEEE Trans. Signal Processing, vol. 39, pp. 2025-2043, Sept. 1991.

[39] J. P. Havlicek, D. S. Harding, N. D. Mamuya, and A. C. Bovik, "Wideband frequency excursions in computed AM-FM image models," in Proc. IEEE Southw. Symp. Image Anal., Interpret., Tucson, AZ, Apr. 5-7, 1998, pp. 211-216.

[40] D. Marr, Vision. New York: Freeman, 1982.

[41] J. E. Marsden and A. J. Tromba, Vector Calculus. San Francisco, CA: Freeman, 1981.

[42] A. V. Oppenheim and R. W. Schafer, Discrete-Time Signal Processing. Englewood Cliffs, NJ: Prentice-Hall, 1989.

[43] G. Hardy, J. E. Littlewood, and G. Pólya, Inequalities, 2nd ed. Cambridge, U.K.: Cambridge Univ. Press, 1952.

[44] I. Selin, "Theory and applications of the notion of complex signal," (in French), RAND Corp., Santa Monica, CA, Tech. Rep. T-92, Aug. 1958. 


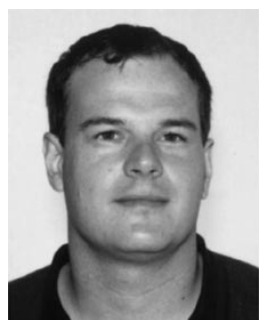

Joseph P. Havlicek (M'88-SM'98) was born in West Lafayette, IN, on April 7, 1964. He received the B.S. degree in 1986 and the M.S. degree in 1988, both from the Virginia Polytechnic Institute and State University, Blacksburg, and the Ph.D. degree in 1996 from the University of Texas, Austin, all in electrical engineering.

From 1984 to 1987, he was a Software Engineer with Management Systems Laboratories, Blacksburg, VA. From 1987 to 1989 , he was with SFA, Inc., Landover, MD, and from 1987 to 1997 he was with the Naval Research Laboratory, Washington, DC, where his work included high-performance signal and image processing. In 1993, he was a Programmer-Analyst with Ralph Kirkley Associates, Austin, working on-site in the multimedia division of IBM, Austin. Since January 1997, he has been an Assistant Professor with the School of Electrical and Computer Engineering, University of Oklahoma, Norman. His research interests include image and video communications, signal processing, and machine vision.

Dr. Havlicek was a recipient of the University of Texas Engineering Foundation Award for Exemplary Engineering Teaching while pursuing a graduate degree in 1992, and the Department of the Navy Award of Merit for Group Achievement in 1990. He is a member of Eta Kappa Nu, Tau Beta Pi, and Phi Kappa Phi.

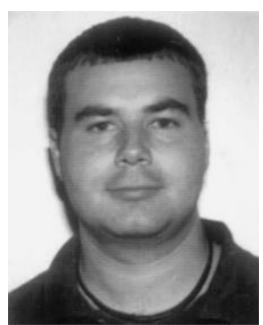

David S. Harding (S'95) was born on August 5, 1974, in Houston, TX. He received the B.S.E. degree with high honors from the University of Texas, Austin, in 1995, where he is currently pursuing the M.S. and Ph.D. degrees.

He was with the Defense Systems and Electronics Group, Texas Instruments, Inc., Dallas. Since 1997, he has been with CyBerCorp, Inc., Austin. His research interests include nonstationary signal analysis, multidimensional signal processing, and computational vision.

Mr. Harding was a recipient of the University of Texas at Austin Microelectronics and Computer Development Fellowship and the Virginia and Ernest Cockrell, Jr. Fellowship in Engineering.

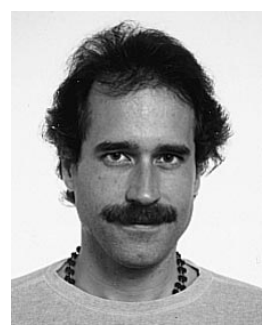

Alan Conrad Bovik (S'80-M'81-SM'89-F'96) was born in Kirkwood, MO, on June 25, 1958. He received the B.S. degree in computer engineering in 1980, and the M.S. and Ph.D. degrees in electrical and computer engineering in 1982 and 1984, respectively, all from the University of Illinois, Urbana-Champaign.

$\mathrm{He}$ is currently the General Dynamics Endowed Fellow and Professor in the Department of Electrical and Computer Engineering, University of Texas, Austin, where he is the Associate Director of the Center for Vision and Image Sciences. During the Spring of 1992, he held a visiting position in the Division of Applied Sciences, Harvard University, Cambridge, MA. His current research interests include digital video, image processing, computer vision, wavelets, three-dimensional microscopy, and computational aspects of biological visual perception. He has published more than 250 technical articles in these areas and holds two U.S. patents.

Dr. Bovik was a recipient of the IEEE Signal Processing Society Meritorious Service Award (1998), the University of Texas Engineering Foundation Halliburton Award, and is a two-time Honorable Mention winner of the International Pattern Recognition Society Award for Outstanding Contribution (1988 and 1993). He has been involved in numerous professional society activities. These currently include Editor-in-Chief, IEEE TRANSACTIONS ON IMAGE PROCESSING, Editorial Board, Proceedings of the IEEE, and Editorial Board, Pattern Recognition. He was the Founding General Chairman of the First IEEE International Conference on Image Processing, Austin, TX, in November, 1994. 